\title{
A System of Coupled Two-sided Sylvester-type Tensor Equations over the Quaternion Algebra
}

\author{
Qing-Wen Wang* and Xiao Wang
}

\begin{abstract}
We establish some necessary and sufficient conditions for the solvability to a system of a pair of coupled two-sided Sylvester-type tensor equations over the quaternion algebra. We also give an expression of the general solution to the system when it is solvable. As applications, we derive some solvability conditions and expressions of the $\eta$-Hermitian solutions to some systems of coupled two-sided Sylvester-type quaternion tensor equations. Moreover, we provide an example to illustrate the main results of this paper.
\end{abstract}

\section{Introduction}

Quaternions was first proposed by Hamilton [14] in 1843. Nowadays quaternions and quaternion matrices have been widely used in many fields such as quantum computing, mechanics, signal and color image processing (e.g., [7, 9, 18, 19, 28, 31, 32, 39]).

Sylvester-type matrix equations have a number of applications in system and control theory, neural network [40, robust control [33], statistics and probability (e.g., [1,20]) and eigenvalue assignment problems 3 . The investigations on Sylvester-type equations over quaternions have attracted more and more attentions in recent years (e.g., [5, 16, 34, 35]).

In recent decades, tensor conceived by Tullio Levi-Civita [21], has attracted a lot of scholars to study, and research results are widely applied in mechanics, data mining, general relativity and so on (e.g., $[2,4,6,10-13,23,27,29,30,36-38]$ ). The two-sided Sylvester-type tensor equation

$$
\mathcal{A} *_{N} \mathcal{X} *_{M} \mathcal{B}+\mathcal{C} *_{N} \mathcal{Y} *_{M} \mathcal{D}=\mathcal{E}
$$

plays an important role in discretization of a linear partial differential equation of high dimension [22]. He, Navasca and Wang 17] investigated the solvability conditions and the expression of the general solution to the equation (1.1) over the quaternion algebra. He 15 defined the $\eta$-Hermitian quaternion tensor as follows. For any $\eta \in\{\mathbf{i}, \mathbf{j}, \mathbf{k}\}$, a Received January 31, 2020; Accepted May 21, 2020.

Communicated by Ren-Cang Li.

2010 Mathematics Subject Classification. 11R52, 15A09, 15A24, 15A69, 15 B33.

Key words and phrases. tensor, Einstein product, tensor equation, quaternion algebra, Moore-Penrose inverse, $\eta$-Hermitian tensor.

*Corresponding author. 
quaternion tensor $\mathcal{A}$ with the order $2 N$ dimension $I_{1} \times \cdots \times I_{N} \times I_{1} \times \cdots \times I_{N}$ is said to be $\eta$-Hermitian if $\mathcal{A}=\mathcal{A}^{\eta^{*}}$, where $\mathcal{A}^{\eta^{*}}=-\eta \mathcal{A}^{*} \eta$, and $\mathcal{A}^{*}$ represents for the conjugate and transpose of $\mathcal{A}$. He also studied the solvability and the general $\eta$-Hermitian solution to a system of quaternion Sylvester-type tensor equations.

To our best knowledge, there has been little information on the two-sided coupled Sylvester-type tensor equations over the quaternion algebra.

Motivated by increasing interest in quaternions, Sylvester-type equations, tensor equations, and in order to improve theoretical understanding of Sylvester-type tensor equations over the quaternion algebra, we in this paper will consider the solvability conditions and the expressions of the solution to the following two systems of coupled two-sided Sylvestertype tensor equations over the quaternion algebra:

$$
\begin{aligned}
& \mathcal{A}_{1} *_{N} \mathcal{X}_{1} *_{M} \mathcal{B}_{1}+\mathcal{C}_{1} *_{N} \mathcal{Y}_{1} *_{M} \mathcal{D}_{1}=\mathcal{E}_{1}, \\
& \mathcal{A}_{2} *_{N} \mathcal{X}_{2} *_{M} \mathcal{B}_{2}+\mathcal{C}_{2} *_{N} \mathcal{Y}_{1} *_{M} \mathcal{D}_{2}=\mathcal{E}_{2},
\end{aligned}
$$

where the operation $*_{N}$ is the Einstein product, and $\mathcal{A}_{i}, \mathcal{B}_{i}, \mathcal{C}_{i}, \mathcal{E}_{i}, \mathcal{D}_{i}(i=1,2)$ are given quaternion tensors and $\mathcal{X}_{i}(i=1,2), \mathcal{Y}_{1}$ are unknown tensors; and

$$
\begin{aligned}
& \mathcal{A}_{1} *_{N} \mathcal{X}_{1} *_{N} \mathcal{A}_{1}^{\eta^{*}}+\mathcal{B}_{1} *_{N} \mathcal{Y}_{1} *_{N} \mathcal{B}_{1}^{\eta^{*}}=\mathcal{E}_{1}, \\
& \mathcal{A}_{2} *_{N} \mathcal{X}_{2} *_{N} \mathcal{A}_{2}^{\eta^{*}}+\mathcal{B}_{2} *_{N} \mathcal{Y}_{1} *_{N} \mathcal{B}_{2}^{\eta^{*}}=\mathcal{E}_{2},
\end{aligned}
$$

where $\mathcal{A}_{i}, \mathcal{B}_{i}, \mathcal{E}_{i}$ are given and $\mathcal{E}_{i}$ are $\eta$-Hermitian quaternion tensors $(i=1,2)$, and $\mathcal{X}_{1}$, $\mathcal{X}_{2}, \mathcal{Y}_{1}$ are unknown $\eta$-Hermitian tensors.

The rest of this paper is organized as follows. In Section 2, we introduce some related definitions and properties about a quaternion tensor. In Section 3, we establish some necessary and sufficient conditions for the existence of a solution to the system 1.2 and give an expression of the general solution when it is solvable. Moreover we present an example to illustrate our results. In Section 4, as applications of the system (1.2), we derive some solvable conditions for the existence of $\eta$-Hermitian solutions to the equation (1.1) and the system (1.3) and give expressions of such solutions when the solvability conditions are met. In Section 5, we give a conclusion to end this paper.

\section{Preliminaries}

Let $\mathbb{R}$ and $\mathbb{H}^{L_{1} \times \cdots \times L_{N}}$ stand, respectively, for the real number field and the set of order $N$ and dimension $L_{1} \times \cdots \times L_{N}$ tensors over the real quaternion algebra

$$
\mathbb{H}=\left\{a_{0}+a_{1} \mathbf{i}+a_{2} \mathbf{j}+a_{3} \mathbf{k} \mid \mathbf{i}^{2}=\mathbf{j}^{2}=\mathbf{k}^{2}=\mathbf{i} \mathbf{j} \mathbf{k}=-1, a_{0}, a_{1}, a_{2}, a_{3} \in \mathbb{R}\right\} .
$$

It is well known that the quaternion algebra is an associative and noncommutative division algebra. For more details about quaternion matrices, please see [28, 39. 
Given $\mathcal{A}=\left(a_{i_{1} \cdots i_{N} j_{1} \cdots j_{M}}\right) \in \mathbb{H}^{I_{1} \times \cdots \times I_{N} \times J_{1} \times \cdots \times J_{M}}$, the tensor $\mathcal{A}^{*}=\left(\bar{a}_{j_{1} \cdots j_{M} i_{1} \cdots i_{N}}\right) \in$ $\mathbb{H}^{J_{1} \times \cdots \times J_{M} \times I_{1} \times \cdots \times I_{N}}$ is called the conjugate transpose of $\mathcal{A}$. A tensor $\mathcal{A} \in \mathbb{H}^{I_{1} \times \cdots \times I_{N} \times I_{1} \times \cdots \times I_{N}}$ is said to be Hermitian if $\mathcal{A}=\mathcal{A}^{*}$. A tensor $\mathcal{D}=\left(d_{i_{1} \cdots i_{N} i_{1} \cdots i_{N}}\right) \in \mathbb{H}^{I_{1} \times \cdots \times I_{N} \times I_{1} \times \cdots \times I_{N}}$ is known as a diagonal tensor if its all entries are zeros except for $d_{i_{1} \cdots i_{N} i_{1} \cdots i_{N}}$. When all the diagonal entries $d_{i_{1} \cdots i_{N} i_{1} \cdots i_{N}}=1, \mathcal{D}$ is the unit tensor.

For $\mathcal{A} \in \mathbb{H}^{I_{1} \times \cdots \times I_{N} \times J_{1} \times \cdots \times J_{N}}$ and $\mathcal{B} \in \mathbb{H}^{J_{1} \times \cdots \times J_{N} \times K_{1} \times \cdots \times K_{M}}$, the Einstein product 8 of the two tensors is defined by the operation $*_{N}$ via

$$
\left(\mathcal{A} *_{N} \mathcal{B}\right)_{i_{1} \cdots i_{N} k_{1} \cdots k_{M}}=\sum_{j_{1} \cdots j_{N}} a_{i_{1} \cdots i_{N} j_{1} \cdots i_{N}} b_{j_{1} \cdots j_{N} k_{1} \cdots k_{M}} .
$$

Hence $\mathcal{A} *_{N} \mathcal{B} \in \mathbb{H}^{I_{1} \times \cdots \times I_{N} \times K_{1} \times \cdots \times K_{M}}$.

Now we introduce the definition of the Moore-Penrose inverse of a tensor over $\mathbb{H}$ via the Einstein product, which is a generation of the Moore-Penrose inverse of a matrix.

Definition 2.1. 17 Let $\mathcal{A} \in \mathbb{H}^{I_{1} \times \cdots \times I_{N} \times J_{1} \times \cdots \times J_{N}}$. The tensor $\mathcal{X} \in \mathbb{H}^{J_{1} \times \cdots \times J_{N} \times I_{1} \times \cdots \times I_{N}}$ satisfying the following four quaternion tensor equalities:

(1) $\mathcal{A} *_{N} \mathcal{X} *_{N} \mathcal{A}=\mathcal{A}$,

(2) $\mathcal{X} *_{N} \mathcal{A} *_{N} \mathcal{X}=\mathcal{X}$

(3) $\left(\mathcal{A} *_{N} \mathcal{X}\right)^{*}=\mathcal{A} *_{N} \mathcal{X}$, and

(4) $\left(\mathcal{X} *_{N} \mathcal{A}\right)^{*}=\mathcal{X} *_{N} \mathcal{A}$

is called the Moore-Penrose inverse of $\mathcal{A}$, denoted by $\mathcal{A}^{\dagger}$.

By 17, for an arbitrary tensor $\mathcal{A} \in \mathbb{H}^{I_{1} \times \cdots \times I_{N} \times J_{1} \times \cdots \times J_{N}}$, the Moore-Penrose inverse exists and is unique. For $\mathcal{C} \in \mathbb{H}^{I_{1} \times \cdots \times I_{N} \times I_{1} \times \cdots \times I_{N}}$, if there is $\mathcal{B} \in \mathbb{H}^{I_{1} \times \cdots \times I_{N} \times I_{1} \times \cdots \times I_{N}}$ satisfying the equation $\mathcal{C} *_{N} \mathcal{B}=\mathcal{B} *_{N} \mathcal{C}=\mathcal{I}$, then $\mathcal{B}$ is regarded as the inverse of $\mathcal{C}$, denoted by $\mathcal{C}^{-1}$. If tensor $\mathcal{C}$ is invertible, then $\mathcal{C}^{\dagger}=\mathcal{C}^{-1}$.

Let the symbols $\mathcal{L}_{\mathcal{A}}$ and $\mathcal{R}_{\mathcal{A}}$ stand for $\mathcal{L}_{\mathcal{A}}=\mathcal{I}-\mathcal{A}^{\dagger} *_{N} \mathcal{A}, \mathcal{R}_{\mathcal{A}}=\mathcal{I}-\mathcal{A} *_{N} \mathcal{A}^{\dagger}$, respectively. Then we have the following properties.

Proposition 2.2. Let $\mathcal{A} \in \mathbb{H}^{I_{1} \times \cdots \times I_{N} \times J_{1} \times \cdots \times J_{N}}$. Then

(1) $\left(\mathcal{A}^{\eta *}\right)^{\dagger}=\left(\mathcal{A}^{\dagger}\right)^{\eta *}$,

(2) $\mathcal{L}_{\mathcal{A}}=\mathcal{L}_{\mathcal{A}}^{*}, \mathcal{R}_{\mathcal{A}}=\mathcal{R}_{\mathcal{A}}^{*}$,

(3) $\mathcal{L}_{\mathcal{A}}^{\eta *}=\mathcal{R}_{\mathcal{A}^{\eta *}}, \mathcal{R}_{\mathcal{A}}^{\eta *}=\mathcal{L}_{\mathcal{A}^{\eta *}}$,

(4) $\left(\mathcal{A}^{\dagger}\right)^{\dagger}=\mathcal{A}$, 
(5) $\left(\mathcal{A}^{*}\right)^{\dagger}=\left(\mathcal{A}^{\dagger}\right)^{*}$,

(6) $\left(\mathcal{A}^{*} *_{N} \mathcal{A}\right)^{\dagger}=\mathcal{A}^{\dagger} *_{N}\left(\mathcal{A}^{*}\right)^{\dagger},\left(\mathcal{A} *_{N} \mathcal{A}^{*}\right)^{\dagger}=\left(\mathcal{A}^{*}\right)^{\dagger} *_{N} \mathcal{A}^{\dagger}$,

(7) $\mathcal{L}_{\mathcal{A}} *_{N} \mathcal{A}^{\dagger}=\mathcal{A} *_{N} \mathcal{L}_{\mathcal{A}}=0, \mathcal{R}_{\mathcal{A}} *_{N} \mathcal{A}=\mathcal{A}^{\dagger} *_{N} \mathcal{R}_{\mathcal{A}}=0$

Now we introduce the block quaternion tensors just as the definitions of block complex tensors $\left[30\right.$. Let $\mathcal{A}=\left(a_{i_{1} \cdots i_{N} j_{1} \cdots j_{M}}\right) \in \mathbb{H}^{I_{1} \times \cdots \times I_{N} \times J_{1} \times \cdots \times J_{M}}$ and $\mathcal{B}=\left(b_{i_{1} \cdots i_{N} k_{1} \cdots k_{M}}\right) \in$ $\mathbb{H}^{I_{1} \times \cdots \times I_{N} \times K_{1} \times \cdots \times K_{M}}$. Then the row block tensor of $\mathcal{A}$ and $\mathcal{B}$ is denoted by

$$
(\mathcal{A} \mathcal{B}) \in \mathbb{H}^{I_{1} \times \cdots \times I_{N} \times L_{1} \times \cdots \times L_{M}},
$$

where $L_{i}=J_{i}+K_{i}, i=1, \ldots, M$ and

$$
(\mathcal{A} \mathcal{B})_{i_{1} \cdots i_{N} l_{1} \cdots l_{M}}= \begin{cases}a_{i_{1} \cdots i_{N} l_{1} \cdots l_{M}} & \text { if } i_{1} \cdots i_{N} \in\left[I_{1}\right] \times \cdots \times\left[I_{N}\right], l_{1} \cdots l_{M} \in\left[J_{1}\right] \times \cdots \times\left[J_{M}\right], \\ b_{i_{1} \cdots i_{N} l_{1} \cdots l_{M}} & \text { if } i_{1} \cdots i_{N} \in\left[I_{1}\right] \times \cdots \times\left[I_{N}\right], l_{1} \cdots l_{M} \in \Gamma_{1} \times \cdots \times \Gamma_{M}, \\ 0 & \text { otherwise }\end{cases}
$$

where $\Gamma_{i}=\left\{J_{i}+1, \ldots, J_{i}+K_{i}\right\}, i=1, \ldots, M$. For given tensors $\mathcal{C}=\left(c_{j_{1} \cdots j_{M} i_{1} \cdots i_{N}}\right) \in$ $\mathbb{H}^{J_{1} \times \cdots \times J_{M} \times I_{1} \times \cdots \times I_{N}}$ and $\mathcal{D}=\left(d_{k_{1} \cdots k_{M} i_{1} \cdots i_{N}}\right) \in \mathbb{H}^{K_{1} \times \cdots \times K_{M} \times I_{1} \times \cdots \times I_{N}}$, the column block tensor of $\mathcal{C}$ and $\mathcal{D}$ is denoted by

$$
\left(\begin{array}{l}
\mathcal{C} \\
\mathcal{D}
\end{array}\right) \in \mathbb{H}^{L_{1} \times \cdots \times L_{M} \times I_{1} \times \cdots \times I_{N}},
$$

where $L_{i}=J_{i}+K_{i}, i=1, \ldots, M$ and

$$
\left(\begin{array}{l}
\mathcal{C} \\
\mathcal{D}
\end{array}\right)_{l_{1} \cdots l_{M} i_{1} \cdots i_{N}}= \begin{cases}c_{l_{1} \cdots l_{M} i_{1} \cdots i_{N}} & \text { if } l_{1} \cdots l_{M} \in\left[J_{1}\right] \times \cdots \times\left[J_{M}\right], i_{1} \cdots i_{N} \in\left[I_{1}\right] \times \cdots \times\left[I_{N}\right], \\
d_{l_{1} \cdots l_{M} i_{1} \cdots i_{N}} & \text { if } l_{1} \cdots l_{M} \in \Gamma_{1} \times \cdots \times \Gamma_{M}, i_{1} \cdots i_{N} \in\left[I_{1}\right] \times \cdots \times\left[I_{N}\right], \\
0 & \text { otherwise, }\end{cases}
$$

where $\Gamma_{i}=\left\{J_{i}+1, \ldots, J_{i}+K_{i}\right\}, i=1, \ldots, M$.

The product of block tensors is given as follows.

Proposition 2.3. Let $(\mathcal{A} \mathcal{B})$ and $\left({ }_{\mathcal{D}}^{\mathcal{C}}\right)$ be of the form in 2.1) and 2.2), respectively. Then

(1) $\mathcal{F} *_{N}(\mathcal{A} \mathcal{B})=\left(\mathcal{F} *_{N} \mathcal{A} \quad \mathcal{F} *_{N} \mathcal{B}\right) \in \mathbb{H}^{I_{1} \times \cdots \times I_{N} \times L_{1} \times \cdots \times L_{M}}$,

(2) $\left(\begin{array}{c}\mathcal{C} \\ \mathcal{D}\end{array}\right) *_{N} \mathcal{F}=\left(\begin{array}{c}\mathcal{C} *_{N} \mathcal{F} \\ \mathcal{D} *_{N} \mathcal{F}\end{array}\right) \in \mathbb{H}^{L_{1} \times \cdots \times L_{M} \times I_{1} \times \cdots \times I_{N}}$,

$(3)(\mathcal{A} \mathcal{B}) *_{M}\left(\begin{array}{l}\mathcal{C} \\ \mathcal{D}\end{array}\right)=\mathcal{A} *_{M} \mathcal{C}+\mathcal{B} *_{M} \mathcal{D} \in \mathbb{H}^{I_{1} \times \cdots \times I_{N} \times I_{1} \times \cdots \times I_{N}}$, where $\mathcal{F} \in \mathbb{H}^{I_{1} \times \cdots \times I_{N} \times I_{1} \times \cdots \times I_{N}}$. 
In order to prove our main result in the next section, we start with the two-sided Sylvester-type quaternion tensor equation (1.1). The following lemma provides the solvability conditions and general solution to the equation (1.1).

Lemma 2.4. [17 Assume that $\mathcal{A} \in \mathbb{H}^{I_{1} \times \cdots \times I_{N} \times J_{1} \times \cdots \times J_{N}}, \mathcal{B} \in \mathbb{H}^{K_{1} \times \cdots \times K_{M} \times L_{1} \times \cdots \times L_{M}}$, $\mathcal{C} \in \mathbb{H}^{I_{1} \times \cdots \times I_{N} \times G_{1} \times \cdots \times G_{N}}, \mathcal{D} \in \mathbb{H}^{H_{1} \times \cdots \times H_{M} \times L_{1} \times \cdots \times L_{M}}$ and $\mathcal{E} \in \mathbb{H}^{I_{1} \times \cdots \times I_{N} \times L_{1} \times \cdots \times L_{M}}$. Set

$$
\mathcal{P}=\mathcal{R}_{\mathcal{A}} *_{N} \mathcal{C}, \quad \mathcal{Q}=\mathcal{D} *_{M} \mathcal{L}_{\mathcal{B}}, \quad \mathcal{S}=\mathcal{C} *_{N} \mathcal{L}_{\mathcal{P}}
$$

Then the equation (1.1) is consistent if and only if

$$
\begin{aligned}
& \mathcal{R}_{\mathcal{P}} *_{N} \mathcal{R}_{\mathcal{A}} *_{N} \mathcal{E}=0, \quad \mathcal{E} *_{M} \mathcal{L}_{\mathcal{B}} *_{M} \mathcal{L}_{\mathcal{Q}}=0, \\
& \mathcal{R}_{\mathcal{A}} *_{N} \mathcal{E} *_{M} \mathcal{L}_{\mathcal{D}}=0, \quad \mathcal{R}_{\mathcal{C}} *_{N} \mathcal{E} *_{M} \mathcal{L}_{\mathcal{B}}=0 .
\end{aligned}
$$

In that case, the general solution to the equation (1.1) can be expressed as follows:

$$
\begin{aligned}
\mathcal{X}= & \mathcal{A}^{\dagger} *_{N} \mathcal{E} *_{M} \mathcal{B}^{\dagger}-\mathcal{A}^{\dagger} *_{N} \mathcal{C} *_{N} \mathcal{P}^{\dagger} *_{N} \mathcal{E} *_{M} \mathcal{B}^{\dagger} \\
& -\mathcal{A}^{\dagger} *_{N} \mathcal{S} *_{N} \mathcal{C}^{\dagger} *_{N} \mathcal{E} *_{M} \mathcal{Q}^{\dagger} *_{M} \mathcal{D} *_{M} \mathcal{B}^{\dagger}-\mathcal{A}^{\dagger} *_{N} \mathcal{S} *_{N} \mathcal{U}_{2} *_{M} \mathcal{R}_{\mathcal{Q}} *_{M} \mathcal{D} *_{M} \mathcal{B}^{\dagger} \\
& +\mathcal{L}_{\mathcal{A}} *_{N} \mathcal{U}_{4}+\mathcal{U}_{5} *_{M} \mathcal{R}_{\mathcal{B}}, \\
\mathcal{Y}= & \mathcal{P}^{\dagger} *_{N} \mathcal{E} *_{M} \mathcal{D}^{\dagger}+\mathcal{S}^{\dagger} *_{N} \mathcal{S} *_{N} \mathcal{C}^{\dagger} *_{N} \mathcal{E} *_{M} \mathcal{Q}^{\dagger}+\mathcal{L}_{\mathcal{P}} *_{N} \mathcal{L}_{\mathcal{S}} *_{N} \mathcal{U}_{1} \\
& +\mathcal{L}_{\mathcal{P}} *_{N} \mathcal{U}_{2} *_{M} \mathcal{R}_{\mathcal{Q}}+\mathcal{U}_{3} *_{M} \mathcal{R}_{\mathcal{D}},
\end{aligned}
$$

where $\mathcal{U}_{1}, \mathcal{U}_{2}, \mathcal{U}_{3}, \mathcal{U}_{4}, \mathcal{U}_{5}$ are arbitrary quaternion tensors with suitable orders.

3. The general solution to the system 1.2

In this section, we consider the solvability conditions and the general solution to 1.2 where $\mathcal{A}_{1} \in \mathbb{H}^{I_{1} \times \cdots \times I_{N} \times J_{1} \times \cdots \times J_{N}}, \mathcal{A}_{2} \in \mathbb{H}^{L_{1} \times \cdots \times L_{N} \times K_{1} \times \cdots \times K_{N}}, \mathcal{C}_{1} \in \mathbb{H}^{I_{1} \times \cdots \times I_{N} \times P_{1} \times \cdots \times P_{N}}$, $\mathcal{C}_{2} \in \mathbb{H}^{L_{1} \times \cdots \times L_{N} \times P_{1} \times \cdots \times P_{N}}, \mathcal{B}_{1} \in \mathbb{H}^{R_{1} \times \cdots \times R_{M} \times S_{1} \times \cdots \times S_{M}}, \mathcal{B}_{2} \in \mathbb{H}^{T_{1} \times \cdots \times T_{M} \times Q_{1} \times \cdots \times Q_{M}}$, $\mathcal{D}_{1} \in \mathbb{H}^{G_{1} \times \cdots \times G_{M} \times S_{1} \times \cdots \times S_{M}}, \mathcal{D}_{2} \in \mathbb{H}^{G_{1} \times \cdots \times G_{M} \times Q_{1} \times \cdots \times Q_{M}}, \mathcal{E}_{1} \in \mathbb{H}^{I_{1} \times \cdots \times I_{N} \times S_{1} \times \cdots \times S_{M}}$, $\mathcal{E}_{2} \in \mathbb{H}^{L_{1} \times \cdots \times L_{N} \times Q_{1} \times \cdots \times Q_{M}}$ are given quaternion tensors. For convenience, we set

$$
\begin{aligned}
& \mathcal{M}_{1}=\mathcal{R}_{\mathcal{A}_{1} *{ }_{N}} \mathcal{C}_{1}, \quad \mathcal{N}_{1}=\mathcal{D}_{1} *_{M} \mathcal{L}_{\mathcal{B}_{1}}, \quad \mathcal{S}_{1}=\mathcal{C}_{1} *_{N} \mathcal{L}_{\mathcal{M}_{1}},
\end{aligned}
$$

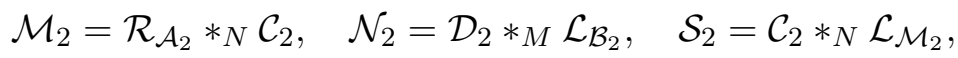

$$
\begin{aligned}
\mathcal{A}_{11}= & \left(\begin{array}{lll}
\mathcal{L}_{\mathcal{M}_{1}} *_{N} & \mathcal{L}_{\mathcal{S}_{1}} & \mathcal{L}_{\mathcal{M}_{2}} *_{N} \mathcal{L}_{\mathcal{S}_{2}}
\end{array}\right), \quad \mathcal{B}_{11}=\left(\begin{array}{c}
\mathcal{R}_{\mathcal{D}_{1}} \\
\mathcal{R}_{\mathcal{D}_{2}}
\end{array}\right), \\
\mathcal{E}_{11}= & \mathcal{M}_{2}^{\dagger} *_{N} \mathcal{E}_{2} *_{M} \mathcal{D}_{2}^{\dagger}+\mathcal{S}_{2}^{\dagger} *_{N} \mathcal{S}_{2} *_{N} \mathcal{C}_{2}^{\dagger} *_{N} \mathcal{E}_{2} *_{M} \mathcal{N}_{2}^{\dagger} \\
& -\mathcal{M}_{1}^{\dagger} *_{N} \mathcal{E}_{1} *_{M} \mathcal{D}_{1}^{\dagger}-\mathcal{S}_{1}^{\dagger} *_{N} \mathcal{S}_{1} *_{N} \mathcal{C}_{1}^{\dagger} *_{N} \mathcal{E}_{1} *_{M} \mathcal{N}_{1}^{\dagger},
\end{aligned}
$$

$$
\begin{aligned}
& \mathcal{A}=\mathcal{R}_{\mathcal{A}_{11} *_{N}} \mathcal{L}_{\mathcal{M}_{1}}, \quad \mathcal{B}=\mathcal{R}_{\mathcal{N}_{1} *_{M}} \mathcal{L}_{\mathcal{B}_{11}}, \quad \mathcal{C}=\mathcal{R}_{\mathcal{A}_{11} *_{N}} \mathcal{L}_{\mathcal{M}_{2}}, \quad \mathcal{D}=\mathcal{R}_{\mathcal{N}_{2}} *_{M} \mathcal{L}_{\mathcal{B}_{11}} \text {, } \\
& \mathcal{E}=\mathcal{R}_{\mathcal{A}_{11}} *_{N} \mathcal{E}_{11} *_{M} \mathcal{L}_{\mathcal{B}_{11}}, \quad \mathcal{M}=\mathcal{R}_{\mathcal{A}} *_{N} \mathcal{C}, \quad \mathcal{N}=\mathcal{D} *_{M} \mathcal{L}_{\mathcal{B}}, \quad \mathcal{S}=\mathcal{C} *_{N} \mathcal{L}_{\mathcal{M}}
\end{aligned}
$$


Theorem 3.1. The system (1.2) is consistent if and only if

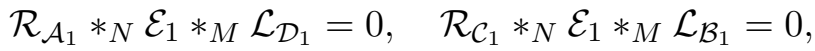

$$
\begin{aligned}
& \mathcal{R}_{\mathcal{M}_{1} *_{N}} \mathcal{R}_{\mathcal{A}_{1} *{ }_{N}} \mathcal{E}_{1}=0, \quad \mathcal{E}_{1} *_{M} \mathcal{L}_{\mathcal{B}_{1}} *_{M} \mathcal{L}_{\mathcal{N}_{1}}=0, \\
& \mathcal{R}_{\mathcal{A}_{2} *_{N}} \mathcal{E}_{2} *_{M} \mathcal{L}_{\mathcal{D}_{2}}=0, \quad \mathcal{R}_{\mathcal{C}_{2}} *_{N} \mathcal{E}_{2} *_{M} \mathcal{L}_{\mathcal{B}_{2}}=0, \\
& \mathcal{R}_{\mathcal{M}_{2}} *_{N} \mathcal{R}_{\mathcal{A}_{2}} *_{N} \mathcal{E}_{2}=0, \quad \mathcal{E}_{2} *_{M} \mathcal{L}_{\mathcal{B}_{2}} *_{M} \mathcal{L}_{\mathcal{N}_{2}}=0, \\
& \mathcal{R}_{\mathcal{M}} *_{N} \mathcal{R}_{\mathcal{A}} *_{N} \mathcal{E}=0, \quad \mathcal{E} *_{M} \mathcal{L}_{\mathcal{B}} *_{M} \mathcal{L}_{\mathcal{N}}=0, \\
& \mathcal{R}_{\mathcal{A}} *_{N} \mathcal{E} *_{M} \mathcal{L}_{\mathcal{D}}=0, \quad \mathcal{R}_{\mathcal{C}} *_{N} \mathcal{E} *_{M} \mathcal{L}_{\mathcal{B}}=0 .
\end{aligned}
$$

In this case, the general solution to the system $(1.2)$ can be expressed as

$$
\begin{aligned}
& \mathcal{X}_{1}=\mathcal{A}_{1}^{\dagger} *_{N} \mathcal{E}_{1} *_{M} \mathcal{B}_{1}^{\dagger}-\mathcal{A}_{1}^{\dagger} *_{N} \mathcal{S}_{1} *_{N} \mathcal{C}_{1}^{\dagger} *_{N} \mathcal{E}_{1} *_{M} \mathcal{N}_{1}^{\dagger} *_{N} \mathcal{D}_{1} *_{M} \mathcal{B}_{1}^{\dagger} \\
& -\mathcal{A}_{1}^{\dagger} *_{N} \mathcal{C}_{1} *_{N} \mathcal{M}_{1}^{\dagger} *_{N} \mathcal{E}_{1} *_{M} \mathcal{B}_{1}^{\dagger}-\mathcal{A}_{1}^{\dagger} *_{N} \mathcal{S}_{1} *_{N} \mathcal{V}_{2} *_{M} \mathcal{R}_{\mathcal{N}_{1}} *_{M} \mathcal{D}_{1} *_{M} \mathcal{B}_{1}^{\dagger} \\
& +\mathcal{L}_{\mathcal{A}_{1} *_{N}} \mathcal{V}_{4}+\mathcal{V}_{5} *_{M} \mathcal{R}_{\mathcal{B}_{1}} \\
& \mathcal{X}_{2}=\mathcal{A}_{2}^{\dagger} *_{N} \mathcal{E}_{2} *_{M} \mathcal{B}_{2}^{\dagger}-\mathcal{A}_{2}^{\dagger} *_{N} \mathcal{S}_{2} *_{N} \mathcal{C}_{2}^{\dagger} *_{N} \mathcal{E}_{2} *_{M} \mathcal{N}_{2}^{\dagger} *_{N} \mathcal{D}_{2} *_{M} \mathcal{B}_{2}^{\dagger} \\
& -\mathcal{A}_{2}^{\dagger} *_{N} \mathcal{C}_{2} *_{N} \mathcal{M}_{2}^{\dagger} *_{N} \mathcal{E}_{2} *_{M} \mathcal{B}_{2}^{\dagger}-\mathcal{A}_{2}^{\dagger} *_{N} \mathcal{S}_{2} *_{N} \mathcal{T}_{2} *_{M} \mathcal{R}_{\mathcal{N}_{2}} *_{M} \mathcal{D}_{2} *_{M} \mathcal{B}_{2}^{\dagger} \\
& +\mathcal{L}_{\mathcal{A}_{2} *_{N}} \mathcal{T}_{4}+\mathcal{T}_{5} *_{M} \mathcal{R}_{\mathcal{B}_{2}}
\end{aligned}
$$

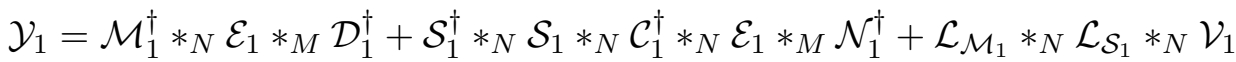

$$
\begin{aligned}
& +\mathcal{L}_{\mathcal{M}_{1}} *_{N} \mathcal{V}_{2} *_{M} \mathcal{R}_{\mathcal{N}_{1}}+\mathcal{V}_{3} *_{M} \mathcal{R}_{\mathcal{D}_{1}}
\end{aligned}
$$

or

$$
\begin{aligned}
\mathcal{Y}_{1}= & \mathcal{M}_{2}^{\dagger} *_{N} \mathcal{E}_{2} *_{M} \mathcal{D}_{2}^{\dagger}+\mathcal{S}_{2}^{\dagger} *_{N} \mathcal{S}_{2} *_{N} \mathcal{C}_{2}^{\dagger} *_{N} \mathcal{E}_{2} *_{M} \mathcal{N}_{2}^{\dagger}-\mathcal{L}_{\mathcal{M}_{2} *_{N} \mathcal{L}_{\mathcal{S}_{2}} *_{N} \mathcal{T}_{1}} \\
& -\mathcal{L}_{\mathcal{M}_{2}} *_{N} \mathcal{T}_{2} *_{M} \mathcal{R}_{\mathcal{N}_{2}}-\mathcal{T}_{3} *_{M} \mathcal{R}_{\mathcal{D}_{2}},
\end{aligned}
$$

where

$$
\begin{aligned}
& \mathcal{V}_{1}=\left(\begin{array}{ll}
\mathcal{I} & 0
\end{array}\right) *_{N}\left(\mathcal{A}_{11}^{\dagger} *_{N}\left(\mathcal{E}_{11}-\mathcal{L}_{\mathcal{M}_{1} *_{N}} \mathcal{V}_{2} *_{M} \mathcal{R}_{\mathcal{N}_{1}}-\mathcal{L}_{\mathcal{M}_{2}} *_{N} \mathcal{T}_{2} *_{M} \mathcal{R}_{\mathcal{N}_{2}}\right)\right. \\
& \left.+\mathcal{W}_{1} *_{M} \mathcal{B}_{11}+\mathcal{L}_{\mathcal{A}_{11}} *_{N} \mathcal{W}_{2}\right) \\
& \mathcal{V}_{2}=\mathcal{A}^{\dagger} *_{N} \mathcal{E} *_{M} \mathcal{B}^{\dagger}-\mathcal{A}^{\dagger} *_{N} \mathcal{S} *_{N} \mathcal{C}^{\dagger} *_{N} \mathcal{E} *_{M} \mathcal{N}^{\dagger} *_{M} \mathcal{D} *_{M} \mathcal{B}^{\dagger} \\
& -\mathcal{A}^{\dagger} *_{N} \mathcal{C} *_{N} \mathcal{M}^{\dagger} *_{N} \mathcal{E} *_{M} \mathcal{B}^{\dagger}-\mathcal{A}^{\dagger} *_{N} \mathcal{S} *_{N} \mathcal{W}_{4} *_{M} \mathcal{R}_{\mathcal{N}} *_{M} \mathcal{D} *_{M} \mathcal{B}^{\dagger} \\
& +\mathcal{L}_{\mathcal{A}} *_{N} \mathcal{W}_{5}+\mathcal{W}_{6} *_{M} \mathcal{R}_{\mathcal{B}} \\
& \mathcal{V}_{3}=\left(\mathcal{R}_{\mathcal{A}_{11}} *_{N}\left(\mathcal{E}_{11}-\mathcal{L}_{\mathcal{M}_{1}} *_{N} \mathcal{V}_{2} *_{M} \mathcal{R}_{\mathcal{N}_{1}}-\mathcal{L}_{\mathcal{M}_{2}} *_{N} \mathcal{T}_{2} *_{M} \mathcal{R}_{\mathcal{N}_{2}}\right) *_{M} \mathcal{B}_{11}^{\dagger}\right. \\
& \left.-\mathcal{A}_{11} *_{N} \mathcal{W}_{1}-\mathcal{W}_{3} *_{M} \mathcal{R}_{\mathcal{B}_{11}}\right) *_{M}\left(\begin{array}{l}
\mathcal{I} \\
0
\end{array}\right) \text {, } \\
& \mathcal{T}_{1}=\left(\begin{array}{ll}
0 & \mathcal{I}
\end{array}\right) *_{N}\left(\mathcal{A}_{11}^{\dagger} *_{N}\left(\mathcal{E}_{11}-\mathcal{L}_{\mathcal{M}_{1} *_{N}} \mathcal{V}_{2} *_{M} \mathcal{R}_{\mathcal{N}_{1}}-\mathcal{L}_{\mathcal{M}_{2} *_{N}} \mathcal{T}_{2} *_{M} \mathcal{R}_{\mathcal{N}_{2}}\right)\right. \\
& \left.+\mathcal{W}_{1} *_{M} \mathcal{B}_{11}+\mathcal{L}_{\mathcal{A}_{11} *_{N}} \mathcal{W}_{2}\right)
\end{aligned}
$$




$$
\begin{aligned}
\mathcal{T}_{2}= & \mathcal{M}^{\dagger} *_{N} \mathcal{E} *_{M} \mathcal{D}^{\dagger}+\mathcal{S} *_{N} \mathcal{S}^{\dagger} *_{N} \mathcal{C}^{\dagger} *_{N} \mathcal{E} *_{M} \mathcal{N}^{\dagger}+\mathcal{L}_{\mathcal{M}} *_{N} \mathcal{L}_{\mathcal{S}} *_{N} \mathcal{W}_{7} \\
& +\mathcal{L}_{\mathcal{M}} *_{N} \mathcal{W}_{4} *_{M} \mathcal{R}_{\mathcal{N}}+\mathcal{W}_{8} *_{M} \mathcal{R}_{\mathcal{D}}, \\
\mathcal{T}_{3}= & \left(\mathcal{R}_{\mathcal{A}_{11} *_{N}}\left(\mathcal{E}_{11}-\mathcal{L}_{\mathcal{M}_{1} *_{N}} \mathcal{V}_{2} *_{M} \mathcal{R}_{\mathcal{N}_{1}}-\mathcal{L}_{\mathcal{M}_{2} *_{N}} \mathcal{T}_{2} *_{M} \mathcal{R}_{\mathcal{N}_{2}}\right) *_{M} \mathcal{B}_{11}^{\dagger}\right. \\
& \left.-\mathcal{A}_{11} *_{N} \mathcal{W}_{1}-\mathcal{W}_{3} *_{M} \mathcal{R}_{\mathcal{B}_{11}}\right) *_{M}\left(\begin{array}{c}
0 \\
\mathcal{I}
\end{array}\right),
\end{aligned}
$$

and $\mathcal{V}_{4}, \mathcal{V}_{5}, \mathcal{T}_{4}, \mathcal{T}_{5}, \mathcal{W}_{1}, \ldots, \mathcal{W}_{8}$ are arbitrary tensors over $\mathbb{H}$ with appropriate sizes.

Proof. We first prove that if the system $\sqrt{1.2}$ has a solution, then the solvable conditions in $3.5-(3.7)$ are true and the general solution can be expressed as $(3.8)-(3.11)$.

If the system 1.2 is solvable, then

$$
\mathcal{A}_{1} *_{N} \mathcal{X}_{1} *_{M} \mathcal{B}_{1}+\mathcal{C}_{1} *_{N} \mathcal{Y}_{1} *_{M} \mathcal{D}_{1}=\mathcal{E}_{1}
$$

and

$$
\mathcal{A}_{2} *_{N} \mathcal{X}_{2} *_{M} \mathcal{B}_{2}+\mathcal{C}_{2} *_{N} \mathcal{Y}_{1} *_{M} \mathcal{D}_{2}=\mathcal{E}_{2}
$$

are consistent. It follows from Lemma 2.4 that the system (3.13) is consistent if and only if (3.5) is satisfied. In this case, the general solution to the system (3.13) can be expressed as

$$
\begin{aligned}
& \mathcal{X}_{1}=\mathcal{A}_{1}^{\dagger} *_{N} \mathcal{E}_{1} *_{M} \mathcal{B}_{1}^{\dagger}-\mathcal{A}_{1}^{\dagger} *_{N} \mathcal{S}_{1} *_{N} \mathcal{C}_{1}^{\dagger} *_{N} \mathcal{E}_{1} *_{M} \mathcal{N}_{1}^{\dagger} *_{N} \mathcal{D}_{1} *_{M} \mathcal{B}_{1}^{\dagger} \\
& -\mathcal{A}_{1}^{\dagger} *_{N} \mathcal{C}_{1} *_{N} \mathcal{M}_{1}^{\dagger} *_{N} \mathcal{E}_{1} *_{M} \mathcal{B}_{1}^{\dagger}-\mathcal{A}_{1}^{\dagger} *_{N} \mathcal{S}_{1} *_{N} \mathcal{V}_{2} *_{M} \mathcal{R}_{\mathcal{N}_{1} *_{M}} \mathcal{D}_{1} *_{M} \mathcal{B}_{1}^{\dagger} \\
& +\mathcal{L}_{\mathcal{A}_{1}} *_{N} \mathcal{V}_{4}+\mathcal{V}_{5} *_{M} \mathcal{R}_{\mathcal{B}_{1}} \text {, } \\
& \mathcal{Y}_{1}=\mathcal{M}_{1}^{\dagger} *_{N} \mathcal{E}_{1} *_{M} \mathcal{D}_{1}^{\dagger}+\mathcal{S}_{1}^{\dagger} *_{N} \mathcal{S}_{1} *_{N} \mathcal{C}_{1}^{\dagger} *_{N} \mathcal{E}_{1} *_{M} \mathcal{N}_{1}^{\dagger}+\mathcal{L}_{\mathcal{M}_{1} *_{N}} \mathcal{L}_{\mathcal{S}_{1}} *_{N} \mathcal{V}_{1} \\
& +\mathcal{L}_{\mathcal{M}_{1}} *_{N} \mathcal{V}_{2} *_{M} \mathcal{R}_{\mathcal{N}_{1}}+\mathcal{V}_{3} *_{M} \mathcal{R}_{\mathcal{D}_{1}}
\end{aligned}
$$

where $\mathcal{V}_{1}, \ldots, \mathcal{V}_{5}$ are arbitrary quaternion tensors with suitable orders. This means $\left(\mathcal{X}_{1}, \mathcal{Y}_{1}\right)$ can be expressed by (3.8) and (3.10).

Similarly, the system (3.14) is consistent if and only if 3.6 is satisfied. Then the general solution can be expressed as

$$
\begin{aligned}
& \mathcal{X}_{2}=\mathcal{A}_{2}^{\dagger} *_{N} \mathcal{E}_{2} *_{M} \mathcal{B}_{2}^{\dagger}-\mathcal{A}_{2}^{\dagger} *_{N} \mathcal{S}_{2} *_{N} \mathcal{C}_{2}^{\dagger} *_{N} \mathcal{E}_{2} *_{M} \mathcal{N}_{2}^{\dagger} *_{N} \mathcal{D}_{2} *_{M} \mathcal{B}_{2}^{\dagger} \\
& -\mathcal{A}_{2}^{\dagger} *_{N} \mathcal{C}_{2} *_{N} \mathcal{M}_{2}^{\dagger} *_{N} \mathcal{E}_{2} *_{M} \mathcal{B}_{2}^{\dagger}-\mathcal{A}_{2}^{\dagger} *_{N} \mathcal{S}_{2} *_{N} \mathcal{T}_{2} *_{M} \mathcal{R}_{\mathcal{N}_{2}} *_{M} \mathcal{D}_{2} *_{M} \mathcal{B}_{2}^{\dagger} \\
& +\mathcal{L}_{\mathcal{A}_{2} *_{N}} \mathcal{T}_{4}+\mathcal{T}_{5} *_{M} \mathcal{R}_{\mathcal{B}_{2}} \\
& \mathcal{Y}_{1}=\mathcal{M}_{2}^{\dagger} *_{N} \mathcal{E}_{2} *_{M} \mathcal{D}_{2}^{\dagger}+\mathcal{S}_{2}^{\dagger} *_{N} \mathcal{S}_{2} *_{N} \mathcal{C}_{2}^{\dagger} *_{N} \mathcal{E}_{2} *_{M} \mathcal{N}_{2}^{\dagger}-\mathcal{L}_{\mathcal{M}_{2}} *_{N} \mathcal{L}_{\mathcal{S}_{2}} *_{N} \mathcal{T}_{1} \\
& -\mathcal{L}_{\mathcal{M}_{2}} *_{N} \mathcal{T}_{2} *_{M} \mathcal{R}_{\mathcal{N}_{2}}-\mathcal{T}_{3} *_{M} \mathcal{R}_{\mathcal{D}_{2}}
\end{aligned}
$$

where $\mathcal{T}_{1}, \ldots, \mathcal{T}_{5}$ are arbitrary tensors over $\mathbb{H}$ with appropriate sizes. This means $\left(\mathcal{X}_{2}, \mathcal{Y}_{1}\right)$ can be expressed by (3.9) and (3.11). 
Equating $\mathcal{Y}_{1}$ in 3.15 and $\mathcal{Y}_{1}$ in 3.16 , we have

$$
\mathcal{A}_{11} *_{N}\left(\begin{array}{l}
\mathcal{V}_{1} \\
\mathcal{T}_{1}
\end{array}\right)+\left(\begin{array}{ll}
\mathcal{V}_{3} & \mathcal{T}_{3}
\end{array}\right) *_{M} \mathcal{B}_{11}=\mathcal{E}_{11}-\mathcal{L}_{\mathcal{M}_{1} *_{N}} \mathcal{V}_{2} *_{M} \mathcal{R}_{\mathcal{N}_{1}}-\mathcal{L}_{\mathcal{M}_{2}} *_{N} \mathcal{T}_{2} *_{M} \mathcal{R}_{\mathcal{N}_{2}}
$$

It follows from Lemma 2.4 that the system (3.17) is consistent if and only if

$$
\mathcal{R}_{\mathcal{A}_{11} *_{N}}\left(\mathcal{E}_{11}-\mathcal{L}_{\mathcal{M}_{1}} *_{N} \mathcal{V}_{2} *_{M} \mathcal{R}_{\mathcal{N}_{1}}-\mathcal{L}_{\mathcal{M}_{2}} *_{N} \mathcal{T}_{2} *_{M} \mathcal{R}_{\mathcal{N}_{2}}\right) *_{M} \mathcal{L}_{\mathcal{B}_{11}}=0
$$

which is equal to

$$
\mathcal{A} *_{N} \mathcal{V}_{2} *_{M} \mathcal{B}+\mathcal{C} *_{N} \mathcal{T}_{2} *_{M} \mathcal{D}=\mathcal{E}
$$

where $\mathcal{A}, \mathcal{B}, \mathcal{C}, \mathcal{D}$ and $\mathcal{E}$ are shown in $(3.3)$ and $(3.4)$. According to Lemma 2.4, the system (3.18) is consistent if and only if (3.7) is satisfied and its general solution can be expressed as

$$
\begin{aligned}
\mathcal{V}_{2}= & \mathcal{A}^{\dagger} *_{N} \mathcal{E} *_{M} \mathcal{B}^{\dagger}-\mathcal{A}^{\dagger} *_{N} \mathcal{S} *_{N} \mathcal{C}^{\dagger} *_{N} \mathcal{E} *_{M} \mathcal{N}^{\dagger} *_{M} \mathcal{D} *_{M} \mathcal{B}^{\dagger} \\
& -\mathcal{A}^{\dagger} *_{N} \mathcal{C} *_{N} \mathcal{M}^{\dagger} *_{N} \mathcal{E} *_{M} \mathcal{B}^{\dagger}-\mathcal{A}^{\dagger} *_{N} \mathcal{S} *_{N} \mathcal{W}_{4} *_{M} \mathcal{R}_{\mathcal{N}} *_{M} \mathcal{D} *_{M} \mathcal{B}^{\dagger} \\
& +\mathcal{L}_{\mathcal{A}} *_{N} \mathcal{W}_{5}+\mathcal{W}_{6} *_{M} \mathcal{R}_{\mathcal{B}}, \\
\mathcal{T}_{2}= & \mathcal{M}^{\dagger} *_{N} \mathcal{E} *_{M} \mathcal{D}^{\dagger}+\mathcal{S} *_{N} \mathcal{S}^{\dagger} *_{N} \mathcal{C}^{\dagger} *_{N} \mathcal{E} *_{M} \mathcal{N}^{\dagger}+\mathcal{L}_{\mathcal{M}} *_{N} \mathcal{L}_{\mathcal{S}} *_{N} \mathcal{W}_{7} \\
& +\mathcal{L}_{\mathcal{M}} *_{N} \mathcal{W}_{4} *_{M} \mathcal{R}_{\mathcal{N}}+\mathcal{W}_{8} *_{M} \mathcal{R}_{\mathcal{D}},
\end{aligned}
$$

where $\mathcal{W}_{4}, \ldots, \mathcal{W}_{8}$ are arbitrary quaternion tensors. Therefore, 3.19 is just as $3.12 \mathrm{~b}$ and 3.20 is the same as $3.12 \mathrm{e})$.

Back to 3.17), we can deduce from Lemma 2.4 that

$$
\begin{aligned}
\left(\begin{array}{c}
\mathcal{V}_{1} \\
\mathcal{T}_{1}
\end{array}\right)= & \mathcal{A}_{11}^{\dagger} *_{N}\left(\mathcal{E}_{11}-\mathcal{L}_{\mathcal{M}_{1} *_{N}} \mathcal{V}_{2} *_{M} \mathcal{R}_{\mathcal{N}_{1}}-\mathcal{L}_{\mathcal{M}_{2} *_{N}} \mathcal{T}_{2} *_{M} \mathcal{R}_{\mathcal{N}_{2}}\right) \\
& +\mathcal{W}_{1} *_{M} \mathcal{B}_{11}+\mathcal{L}_{\mathcal{A}_{11} *_{N}} \mathcal{W}_{2}, \\
\left(\mathcal{V}_{3} \mathcal{T}_{3}\right)= & \mathcal{R}_{\mathcal{A}_{11} *_{N}}\left(\mathcal{E}_{11}-\mathcal{L}_{\mathcal{M}_{1} *_{N}} \mathcal{V}_{2} *_{M} \mathcal{R}_{\mathcal{N}_{1}}-\mathcal{L}_{\mathcal{M}_{2} *_{N}} \mathcal{T}_{2} *_{M} \mathcal{R}_{\mathcal{N}_{2}}\right) *_{M} \mathcal{B}_{11}^{\dagger} \\
& -\mathcal{A}_{11} *_{N} \mathcal{W}_{1}-\mathcal{W}_{3} *_{M} \mathcal{R}_{\mathcal{B}_{11}},
\end{aligned}
$$

where $\mathcal{W}_{1}, \mathcal{W}_{2}, \mathcal{W}_{3}$ are arbitrary quaternion tensors with suitable sizes.

By applying Proposition 2.3, we obtain that

$$
\begin{aligned}
& \mathcal{V}_{1}=\left(\begin{array}{ll}
\mathcal{I} & 0
\end{array}\right) *_{N}\left(\mathcal{A}_{11}^{\dagger} *_{N}\left(\mathcal{E}_{11}-\mathcal{L}_{\mathcal{M}_{1}} *_{N} \mathcal{V}_{2} *_{M} \mathcal{R}_{\mathcal{N}_{1}}-\mathcal{L}_{\mathcal{M}_{2}} *_{N} \mathcal{T}_{2} *_{M} \mathcal{R}_{\mathcal{N}_{2}}\right)\right. \\
& \left.+\mathcal{W}_{1} *_{M} \mathcal{B}_{11}+\mathcal{L}_{\mathcal{A}_{11} *_{N}} \mathcal{W}_{2}\right) \\
& \mathcal{V}_{3}=\left(\mathcal{R}_{\mathcal{A}_{11}} *_{N}\left(\mathcal{E}_{11}-\mathcal{L}_{\mathcal{M}_{1}} *_{N} \mathcal{V}_{2} *_{M} \mathcal{R}_{\mathcal{N}_{1}}-\mathcal{L}_{\mathcal{M}_{2}} *_{N} \mathcal{T}_{2} *_{M} \mathcal{R}_{\mathcal{N}_{2}}\right) *_{M} \mathcal{B}_{11}^{\dagger}\right.
\end{aligned}
$$




$$
\begin{aligned}
& \left.-\mathcal{A}_{11} *_{N} \mathcal{W}_{1}-\mathcal{W}_{3} *_{M} \mathcal{R}_{\mathcal{B}_{11}}\right) *_{M}\left(\begin{array}{l}
\mathcal{I} \\
0
\end{array}\right), \\
& \mathcal{T}_{1}=\left(\begin{array}{ll}
0 & \mathcal{I}
\end{array}\right) *_{N}\left(\mathcal{A}_{11}^{\dagger} *_{N}\left(\mathcal{E}_{11}-\mathcal{L}_{\mathcal{M}_{1}} *_{N} \mathcal{V}_{2} *_{M} \mathcal{R}_{\mathcal{N}_{1}}-\mathcal{L}_{\mathcal{M}_{2}} *_{N} \mathcal{T}_{2} *_{M} \mathcal{R}_{\mathcal{N}_{2}}\right)\right. \\
& \left.+\mathcal{W}_{1} *_{M} \mathcal{B}_{11}+\mathcal{L}_{\mathcal{A}_{11} *_{N}} \mathcal{W}_{2}\right), \\
& \mathcal{T}_{3}=\left(\mathcal{R}_{\mathcal{A}_{11} *_{N}}\left(\mathcal{E}_{11}-\mathcal{L}_{\mathcal{M}_{1}} *_{N} \mathcal{V}_{2} *_{M} \mathcal{R}_{\mathcal{N}_{1}}-\mathcal{L}_{\mathcal{M}_{2}} *_{N} \mathcal{T}_{2} *_{M} \mathcal{R}_{\mathcal{N}_{2}}\right) *_{M} \mathcal{B}_{11}^{\dagger}\right. \\
& \left.-\mathcal{A}_{11} *_{N} \mathcal{W}_{1}-\mathcal{W}_{3} *_{M} \mathcal{R}_{\mathcal{B}_{11}}\right) *_{M}\left(\begin{array}{l}
0 \\
\mathcal{I}
\end{array}\right) \text {. }
\end{aligned}
$$

Therefore, $\mathcal{V}_{1}, \mathcal{V}_{3}, \mathcal{T}_{1}, \mathcal{T}_{3}$ can be expressed by (3.12a), (3.12c), 3.12d), 3.12f), respectively.

Now we show that tensors $\mathcal{X}_{i}(i=1,2), \mathcal{Y}_{1}$ having the form of $(3.8)-(3.11)$, respectively, constitute a set of solution to the system (1.2) under the conditions (3.5)-(3.7).

If 3.5 is satisfied, then we can deduced from Lemma 2.4 that 3.8 and 3.10 are solutions to the system (3.13). Similarly, we know that 3.9 and $(3.11$ are a set of solutions to the system 3.14 if 3.6 is met.

Now we show that $\mathcal{Y}_{1}$ in 3.10 and $\mathcal{Y}_{1}$ in 3.11 are equal, that is

$$
\begin{aligned}
& \mathcal{M}_{1}^{\dagger} *_{N} \mathcal{E}_{1} *_{M} \mathcal{D}_{1}^{\dagger}+\mathcal{S}_{1}^{\dagger} *_{N} \mathcal{S}_{1} *_{N} \mathcal{C}_{1}^{\dagger} *_{N} \mathcal{E}_{1} *_{M} \mathcal{N}_{1}^{\dagger}+\mathcal{L}_{\mathcal{M}_{1} *_{N}} \mathcal{L}_{\mathcal{S}_{1} *_{N}} \mathcal{V}_{1} \\
& +\mathcal{L}_{\mathcal{M}_{1}} *_{N} \mathcal{V}_{2} *_{M} \mathcal{R}_{\mathcal{N}_{1}}+\mathcal{V}_{3} *_{M} \mathcal{R}_{\mathcal{D}_{1}} \\
& =\mathcal{M}_{2}^{\dagger} *_{N} \mathcal{E}_{2} *_{M} \mathcal{D}_{2}^{\dagger}+\mathcal{S}_{2}^{\dagger} *_{N} \mathcal{S}_{2} *_{N} \mathcal{C}_{2}^{\dagger} *_{N} \mathcal{E}_{2} *_{M} \mathcal{N}_{2}^{\dagger}-\mathcal{L}_{\mathcal{M}_{2}} *_{N} \mathcal{L}_{\mathcal{S}_{2}} *_{N} \mathcal{T}_{1} \\
& -\mathcal{L}_{\mathcal{M}_{2}} *_{N} \mathcal{T}_{2} *_{M} \mathcal{R}_{\mathcal{N}_{2}}-\mathcal{T}_{3} *_{M} \mathcal{R}_{\mathcal{D}_{2}} \text {. }
\end{aligned}
$$

Using (3.1) and (3.2), we can simplify (3.23) into the form as in (3.17). So we need to show that (3.17) is true. Substituting (3.21) and (3.22) into (3.17) yields

$$
\begin{aligned}
& \mathcal{A}_{11} *_{N}\left(\mathcal{A}_{11}^{\dagger} *_{N}\left(\mathcal{E}_{11}-\mathcal{L}_{\mathcal{M}_{1} *_{N}} \mathcal{V}_{2} *_{M} \mathcal{R}_{\mathcal{N}_{1}}-\mathcal{L}_{\mathcal{M}_{2} *_{N}} \mathcal{T}_{2} *_{M} \mathcal{R}_{\mathcal{N}_{2}}\right)+\mathcal{W}_{1} *_{M} \mathcal{B}_{11}+\mathcal{L}_{\mathcal{A}_{11} *_{N}} \mathcal{W}_{2}\right) \\
& +\left(\mathcal{R}_{\mathcal{A}_{11} *_{N}}\left(\mathcal{E}_{11}-\mathcal{L}_{\mathcal{M}_{1} *_{N}} \mathcal{V}_{2} *_{M} \mathcal{R}_{\mathcal{N}_{1}}-\mathcal{L}_{\mathcal{M}_{2}} *_{N} \mathcal{T}_{2} *_{M} \mathcal{R}_{\mathcal{N}_{2}}\right) *_{M} \mathcal{B}_{11}^{\dagger}\right. \\
& \left.-\mathcal{A}_{11} *_{N} \mathcal{W}_{1}-\mathcal{W}_{3} *_{M} \mathcal{R}_{\mathcal{B}_{11}}\right) *_{M} \mathcal{B}_{11} \\
& =\mathcal{R}_{\mathcal{A}_{11} *_{N}}\left(\mathcal{E}_{11}-\mathcal{L}_{\mathcal{M}_{1}} *_{N} \mathcal{V}_{2} *_{M} \mathcal{R}_{\mathcal{N}_{1}}-\mathcal{L}_{\mathcal{M}_{2} *_{N}} \mathcal{T}_{2} *_{M} \mathcal{R}_{\mathcal{N}_{2}}\right) *_{M} \mathcal{L}_{\mathcal{B}_{11}} \\
& +\mathcal{E}_{11}-\mathcal{L}_{\mathcal{M}_{1}} *_{N} \mathcal{V}_{2} *_{M} \mathcal{R}_{\mathcal{N}_{1}}-\mathcal{L}_{\mathcal{M}_{2}} *_{N} \mathcal{T}_{2} *_{M} \mathcal{R}_{\mathcal{N}_{2}} \\
& =\mathcal{E}-\mathcal{A} *_{N} \mathcal{V}_{2} *_{M} \mathcal{B}-\mathcal{C} *_{N} \mathcal{T}_{2} *_{M} \mathcal{D}+\mathcal{E}_{11}-\mathcal{L}_{\mathcal{M}_{1}} *_{N} \mathcal{V}_{2} *_{M} \mathcal{R}_{\mathcal{N}_{1}}-\mathcal{L}_{\mathcal{M}_{2}} *_{N} \mathcal{T}_{2} *_{M} \mathcal{R}_{\mathcal{N}_{2}} \text {. }
\end{aligned}
$$

Since 3.7 is satisfied, it follows from Lemma 2.4 that $\mathcal{V}_{2}$ in 3.19 and $\mathcal{T}_{2}$ in 3.20 constitute a solution to the system (3.18), that is to say

$$
\mathcal{E}-\mathcal{A} *_{N} \mathcal{V}_{2} *_{M} \mathcal{B}-\mathcal{C} *_{N} \mathcal{T}_{2} *_{M} \mathcal{D}=0
$$

where $\mathcal{V}_{2}$ and $\mathcal{T}_{2}$, respectively, are the form of (3.19) and 3.20). Then (3.17) is satisfied. Therefore, $\mathcal{Y}_{1}$ in $(3.9)$ and $\mathcal{Y}_{1}$ in (3.11) are equal. This means that tensors $\mathcal{X}_{i}, \mathcal{Y}_{1}(i=1,2)$ as the form of (3.8)-(3.11), respectively, constitute a solution to the system 1.2 when (3.5)- (3.7) are satisfied. 
We now give an example to illustrate our results.

Example 3.2. For the system $(1.2), \mathcal{A}_{i}, \mathcal{B}_{i}, \mathcal{C}_{i}, \mathcal{D}_{i}$ and $\mathcal{E}_{i}(i=1,2)$ are quaternion tensors given by

$$
\begin{aligned}
& \mathcal{A}_{1}(:,:, 1,1)=\left(\begin{array}{cc}
\mathbf{i} & \mathbf{j} \\
-\mathbf{i} & -\mathbf{j}
\end{array}\right), \\
& \mathcal{A}_{1}(:,:, 1,2)=\left(\begin{array}{c}
\mathbf{i}-\mathbf{j}-\mathbf{i} \\
\mathbf{i}+\mathbf{k}-\mathbf{j}
\end{array}\right), \\
& \mathcal{A}_{1}(:,:, 2,1)=\left(\begin{array}{cc}
\mathbf{i}+\mathbf{k}-\mathbf{k} \\
\mathbf{i}+\mathbf{j}-\mathbf{j}
\end{array}\right), \\
& \mathcal{A}_{1}(:,:, 2,2)=\left(\begin{array}{cc}
1+\mathbf{i} & 1+\mathbf{j} \\
\mathbf{i} & -\mathbf{k}
\end{array}\right), \\
& \mathcal{A}_{2}(:,:, 1,1)=\left(\begin{array}{cc}
\mathbf{i}+\mathbf{j}-\mathbf{k} & \mathbf{i}+\mathbf{k} \\
\mathbf{j} & \mathbf{i}+\mathbf{j}
\end{array}\right), \\
& \mathcal{A}_{2}(:,:, 1,2)=\left(\begin{array}{cc}
2 \mathbf{i}+\mathbf{j} & -\mathbf{i} \\
\mathbf{j}+\mathbf{k} & \mathbf{i}
\end{array}\right), \\
& \mathcal{A}_{2}(:,:, 2,1)=\left(\begin{array}{cc}
-2 \mathbf{i}+\mathbf{k} & \mathbf{i}-\mathbf{j} \\
1+\mathbf{i} & \mathbf{i}+\mathbf{k}
\end{array}\right), \\
& \mathcal{A}_{2}(:,:, 2,2)=\left(\begin{array}{cc}
\mathbf{i}-\mathbf{j} & 1+\mathbf{k} \\
\mathbf{j}-\mathbf{k} & \mathbf{i}+\mathbf{j}
\end{array}\right), \\
& \mathcal{B}_{1}(:,:, 1,2)=\left(\begin{array}{cc}
1-\mathbf{i} & \mathbf{j} \\
\mathbf{i}+\mathbf{k} & -\mathbf{k}
\end{array}\right), \\
& \mathcal{B}_{1}(:,:, 2,1)=\left(\begin{array}{l}
\mathbf{j}-\mathbf{k}-\mathbf{i} \\
\mathbf{i}+\mathbf{k}-\mathbf{j}
\end{array}\right), \\
& \mathcal{B}_{1}(:,:, 1,1)=\left(\begin{array}{cc}
\mathbf{j}-\mathbf{k} & 0 \\
0 & \mathbf{i}-\mathbf{k}
\end{array}\right), \\
& \mathcal{B}_{2}(:,:, 1,1)=\left(\begin{array}{cc}
\mathbf{i}-\mathbf{k} & \mathbf{i}+\mathbf{k} \\
\mathbf{j} & 1-\mathbf{i}
\end{array}\right), \\
& \mathcal{B}_{2}(:,:, 1,2)=\left(\begin{array}{cc}
\mathbf{i}+\mathbf{j} & -\mathbf{i}+\mathbf{k} \\
1+\mathbf{j} & -\mathbf{i}
\end{array}\right), \\
& \mathcal{B}_{1}(:,:, 2,2)=\left(\begin{array}{cc}
-\mathbf{i} & 0 \\
\mathbf{j} & 0
\end{array}\right), \\
& \mathcal{B}_{2}(:,:, 2,2)=\left(\begin{array}{cc}
\mathbf{j}-\mathbf{k} & 0 \\
\mathbf{i}+\mathbf{j} & 2 \mathbf{j}
\end{array}\right), \\
& \mathcal{C}_{1}(:,:, 1,1)=\left(\begin{array}{cc}
1 & 1+\mathbf{k} \\
\mathbf{i}-\mathbf{k} & \mathbf{j}
\end{array}\right), \\
& \mathcal{B}_{2}(:,:, 2,1)=\left(\begin{array}{cc}
\mathbf{i} & \mathbf{i}-\mathbf{j} \\
\mathbf{i} \mathbf{i}-\mathbf{k}
\end{array}\right), \\
& \mathcal{C}_{1}(:,:, 2,1)=\left(\begin{array}{cc}
-\mathbf{j}+\mathbf{k} & \mathbf{i}-\mathbf{k} \\
\mathbf{j} & 1+\mathbf{j}
\end{array}\right), \\
& \mathcal{C}_{1}(:,:, 2,2)=\left(\begin{array}{ccc}
\mathbf{i}-\mathbf{k} & -\mathbf{j} \\
\mathbf{j}+\mathbf{k} & \mathbf{i}-\mathbf{k}
\end{array}\right),
\end{aligned}
$$

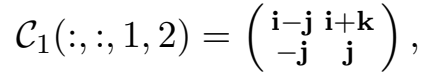

$$
\begin{aligned}
& \mathcal{C}_{2}(:,:, 1,2)=\left(\begin{array}{cc}
\mathbf{i}-\mathbf{k} & -1+\mathbf{j} \\
-\mathbf{k} & 1+\mathbf{i}-\mathbf{j}
\end{array}\right), \\
& \mathcal{C}_{2}(:,:, 2,1)=\left(\begin{array}{c}
1-\mathbf{j} 1+\mathbf{k} \\
1+\mathbf{j} \mathbf{i}+\mathbf{k}
\end{array}\right), \\
& \mathcal{C}_{2}(:,:, 1,1)=\left(\begin{array}{cc}
\mathbf{i}+\mathbf{j} & 1+\mathbf{i} \\
\mathbf{i}-\mathbf{k} & 1-\mathbf{j}
\end{array}\right), \\
& \mathcal{D}_{1}(:,:, 1,1)=\left(\begin{array}{cc}
\mathbf{i}+\mathbf{j} & 1 \\
1+\mathbf{i} & \mathbf{i}
\end{array}\right), \\
& \mathcal{D}_{1}(:,:, 1,2)=\left(\begin{array}{cc}
\mathbf{i}-\mathbf{k} & \mathbf{j} \\
\mathbf{j} & \mathbf{i}+\mathbf{k}
\end{array}\right) \text {, } \\
& \mathcal{C}_{2}(:,:, 2,2)=\left(\begin{array}{cc}
1+\mathbf{i} & 1-\mathbf{i} \\
-\mathbf{i}-\mathbf{k} & \mathbf{j}-\mathbf{k}
\end{array}\right), \\
& \mathcal{D}_{1}(:,:, 2,2)=\left(\begin{array}{cc}
\mathbf{i}+\mathbf{k} & \mathbf{i} \\
\mathbf{j} & -\mathbf{k}
\end{array}\right) \text {, } \\
& \mathcal{D}_{2}(:,:, 1,1)=\left(\begin{array}{cc}
\mathbf{i}+\mathbf{j} & \mathbf{i}-\mathbf{k} \\
-1+\mathbf{j} & 1+2 \mathbf{j}
\end{array}\right), \\
& \mathcal{D}_{2}(:,:, 2,1)=\left(\begin{array}{cc}
-1-\mathbf{i} & \mathbf{k} \\
-\mathbf{i}+\mathbf{k} & 1+\mathbf{k}
\end{array}\right), \\
& \mathcal{D}_{2}(:,:, 2,2)=\left(\begin{array}{c}
\mathbf{i}+2 \mathbf{j} \\
1+\mathbf{j}+\mathbf{k} \\
\mathbf{j}-2 \mathbf{k}
\end{array}\right), \\
& \mathcal{E}_{1}(:,:, 1,1)=\left(\begin{array}{cc}
-2 \mathbf{i}+\mathbf{k} & -3+4 \mathbf{j}-7 \mathbf{k} \\
-1+3 \mathbf{i}+4 \mathbf{j}-4 \mathbf{k} & 7-4 \mathbf{i}+3 \mathbf{j}-6 \mathbf{k}
\end{array}\right), \\
& \mathcal{E}_{1}(:,:, 1,2)=\left(\begin{array}{cc}
-3-14 \mathbf{i}-2 \mathbf{j}+4 \mathbf{k} & -3+3 \mathbf{i}-5 \mathbf{j}-6 \mathbf{k} \\
-7-2 \mathbf{i}-\mathbf{k} & 9+3 \mathbf{i}+\mathbf{j}
\end{array}\right), \\
& \mathcal{E}_{1}(:,:, 2,1)=\left(\begin{array}{cc}
-9+4 \mathbf{i}+5 \mathbf{j}-6 \mathbf{k} & 4-8 \mathbf{i}+2 \mathbf{j}-8 \mathbf{k} \\
10 \mathbf{i}-6 \mathbf{j}-4 \mathbf{k} & -1-\mathbf{i}-10 \mathbf{j}-\mathbf{k}
\end{array}\right), \\
& \mathcal{E}_{1}(:,:, 2,2)=\left(\begin{array}{cc}
1-10 \mathbf{i}-\mathbf{j}+3 \mathbf{k} & 4-2 \mathbf{i}+8 \mathbf{j}+6 \mathbf{k} \\
-7+2 \mathbf{i}-4 \mathbf{j}-\mathbf{k} & -5 \mathbf{i}-\mathbf{j}+11 \mathbf{k}
\end{array}\right), \\
& \mathcal{E}_{2}(:,:, 1,1)=\left(\begin{array}{cc}
-20-4 \mathbf{i}-12 \mathbf{j}+8 \mathbf{k} & 3-2 \mathbf{i}+16 \mathbf{j}-5 \mathbf{k} \\
-1+10 \mathbf{i}-7 \mathbf{j}-4 \mathbf{k} & -16-2 \mathbf{i}-8 \mathbf{j}-6 \mathbf{k}
\end{array}\right), \\
& \mathcal{E}_{2}(:,:, 1,2)=\left(\begin{array}{cc}
10-13 \mathbf{j}+4 \mathbf{k} & -14-5 \mathbf{i}+6 \mathbf{j}+11 \mathbf{k} \\
-4+14 \mathbf{i}+7 \mathbf{j}+7 \mathbf{k} & -11+13 \mathbf{i}-13 \mathbf{j}
\end{array}\right), \\
& \mathcal{E}_{2}(:,:, 2,1)=\left(\begin{array}{cc}
19+6 \mathbf{i}-12 \mathbf{j}-11 \mathbf{k} & -4-5 \mathbf{i}+5 \mathbf{j} \\
-2-13 \mathbf{i}+5 \mathbf{j}-9 \mathbf{k} & 13-9 \mathbf{i}+14 \mathbf{j}-\mathbf{k}
\end{array}\right), \\
& \mathcal{E}_{2}(:,:, 2,2)=\left(\begin{array}{cc}
-3-2 \mathbf{i}-12 \mathbf{j}-\mathbf{k} & 5 \mathbf{j}+9 \mathbf{k} \\
-5+6 \mathbf{i}+2 \mathbf{j}+15 \mathbf{k} & -6-3 \mathbf{i}-9 \mathbf{j}+6 \mathbf{k}
\end{array}\right) .
\end{aligned}
$$

Direct computation yields

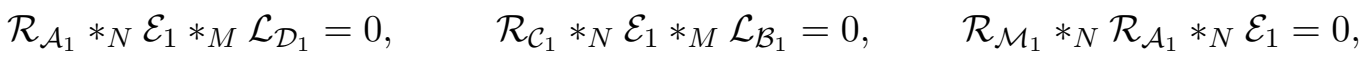

$$
\begin{aligned}
& \mathcal{E}_{1} *_{M} \mathcal{L}_{\mathcal{B}_{1}} *_{M} \mathcal{L}_{\mathcal{N}_{1}}=0, \quad \mathcal{R}_{\mathcal{A}_{2}} *_{N} \mathcal{E}_{2} *_{M} \mathcal{L}_{\mathcal{D}_{2}}=0, \quad \mathcal{R}_{\mathcal{C}_{2}} *_{N} \mathcal{E}_{2} *_{M} \mathcal{L}_{\mathcal{B}_{2}}=0, \\
& \mathcal{R}_{\mathcal{M}_{2}} *_{N} \mathcal{R}_{\mathcal{A}_{2} *_{N}} \mathcal{E}_{2}=0, \quad \mathcal{E}_{2} *_{M} \mathcal{L}_{\mathcal{B}_{2}} *_{M} \mathcal{L}_{\mathcal{N}_{2}}=0, \quad \mathcal{R}_{\mathcal{M}} *_{N} \mathcal{R}_{\mathcal{A}} *_{N} \mathcal{E}=0, \\
& \mathcal{E} *_{M} \mathcal{L}_{\mathcal{B}} *_{M} \mathcal{L}_{\mathcal{N}}=0, \quad \mathcal{R}_{\mathcal{A}} *_{N} \mathcal{E} *_{M} \mathcal{L}_{\mathcal{D}}=0, \quad \mathcal{R}_{\mathcal{C}} *_{N} \mathcal{E} *_{M} \mathcal{L}_{\mathcal{B}}=0 .
\end{aligned}
$$

Therefore, all conditions in Theorem 3.1 are met, the system 1.2 is consistent. As a consequence, it is easy to verify that $\mathcal{X}_{1}, \mathcal{X}_{2}, \mathcal{Y}_{1}$ below satisfy the system $(1.2)$ :

$$
\begin{array}{lll}
\mathcal{X}_{1}(:,:, 1,1)=\left(\begin{array}{cc}
\mathbf{i}-\mathbf{k} \mathbf{j}+\mathbf{k} \\
-\mathbf{k} & 1-\mathbf{i}
\end{array}\right), & \mathcal{X}_{1}(:,:, 1,2)=\left(\begin{array}{cc}
-\mathbf{j}+\mathbf{k} & 1+\mathbf{i} \\
\mathbf{j} & 1-\mathbf{i}
\end{array}\right), & \mathcal{X}_{1}(:,:, 2,1)=\left(\begin{array}{l}
1+\mathbf{i} \mathbf{i}-\mathbf{j} \\
1-\mathbf{i} \mathbf{i}+\mathbf{j}
\end{array}\right), \\
\mathcal{X}_{1}(:,:, 2,2)=\left(\begin{array}{cc}
\mathbf{i} \mathbf{i}-\mathbf{k} \\
\mathbf{i}+\mathbf{j} & \mathbf{j}
\end{array}\right), & \mathcal{X}_{2}(:,:, 1,1)=\left(\begin{array}{cc}
\mathbf{i}-\mathbf{k} \mathbf{i}-\mathbf{j} \\
\mathbf{j}-\mathbf{k} & 1-\mathbf{j}
\end{array}\right), & \mathcal{X}_{2}(:,:, 1,2)=\left(\begin{array}{l}
1+\mathbf{j} \mathbf{i}-\mathbf{k} \\
\mathbf{i}+\mathbf{k} 1-\mathbf{k}
\end{array}\right),
\end{array}
$$




$$
\begin{array}{lll}
\mathcal{X}_{2}(:,:, 2,1)=\left(\begin{array}{cc}
\mathbf{i}+\mathbf{k} & \mathbf{j}-\mathbf{k} \\
\mathbf{i}+\mathbf{j} & -1+\mathbf{j}
\end{array}\right), & \mathcal{X}_{2}(:,:, 2,2)=\left(\begin{array}{cc}
-\mathbf{j} & \mathbf{i}+\mathbf{j} \\
\mathbf{j}-\mathbf{k} & 2 \mathbf{j}
\end{array}\right), & \mathcal{Y}_{1}(:,:, 1,1)=\left(\begin{array}{cc}
1-\mathbf{i} & \mathbf{i}-\mathbf{k} \\
-1-2 \mathbf{j}+\mathbf{i}
\end{array}\right), \\
\mathcal{Y}_{1}(:,:, 1,2)=\left(\begin{array}{cc}
-\mathbf{i}+\mathbf{j} & \mathbf{i} \\
\mathbf{i}-\mathbf{k} & \mathbf{i}+\mathbf{k}
\end{array}\right), & \mathcal{Y}_{1}(:,:, 2,1)=\left(\begin{array}{cc}
1-\mathbf{j} & -\mathbf{k} \\
1-\mathbf{k} & \mathbf{i}
\end{array}\right), & \mathcal{Y}_{1}(:,:, 2,2)=\left(\begin{array}{c}
\mathbf{i} \mathbf{j}-\mathbf{k} \\
\mathbf{j} 1-\mathbf{k}
\end{array}\right) .
\end{array}
$$

4. The $\eta$-Hermitian solutions to the tensor equation (1.1) and the system (1.3)

In this section, we investigate the $\eta$-Hermitian solutions to the equation (1.1) and the system (1.3) based on Theorem 3.1.

We first consider the equation (1.1) where $\mathcal{X}, \mathcal{Y}$ are unknown quaternion tensors with $\mathcal{Y}=\mathcal{Y}^{\eta^{*}}$, and $\mathcal{A} \in \mathbb{H}^{I_{1} \times \cdots \times I_{N} \times J_{1} \times \cdots \times J_{N}}, \mathcal{B} \in \mathbb{H}^{K_{1} \times \cdots \times K_{M} \times R_{1} \times \cdots \times R_{M}}, \mathcal{C} \in \mathbb{H}^{I_{1} \times \cdots \times I_{N} \times P_{1} \times \cdots \times P_{N}}$, $\mathcal{D} \in \mathbb{H}^{P_{1} \times \cdots \times P_{N} \times R_{1} \times \cdots \times R_{M}}, \mathcal{E} \in \mathbb{H}^{I_{1} \times \cdots \times I_{N} \times R_{1} \times \cdots \times R_{M}}$ are given. For simplicity, we put

$$
\begin{aligned}
& \mathcal{M}_{1}=\mathcal{R}_{\mathcal{A}} *_{N} \mathcal{C}, \quad \mathcal{N}_{1}=\mathcal{D} *_{M} \mathcal{L}_{\mathcal{B}}, \quad \mathcal{S}_{1}=\mathcal{C} *_{N} \mathcal{L}_{\mathcal{M}_{1}}, \quad \mathcal{S}_{2}=\left(\mathcal{R}_{\mathcal{N}_{1}} *_{N} \mathcal{D}\right)^{\eta *},
\end{aligned}
$$

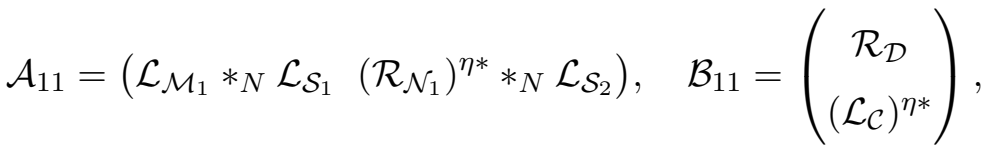

$$
\begin{aligned}
& \mathcal{E}_{11}=\left(\mathcal{N}_{1}^{\dagger}\right)^{\eta *} *_{M} \mathcal{E}^{\eta *} *_{N}\left(\mathcal{C}^{\dagger}\right)^{\eta *}+\mathcal{S}_{2}^{\dagger} *_{M} \mathcal{S}_{2} *_{N}\left(\mathcal{D}^{\dagger}\right)^{\eta *} *_{M} \mathcal{E}^{\eta *} *_{N}\left(\mathcal{M}_{1}^{\dagger}\right)^{\eta *} \\
& -\mathcal{M}_{1}^{\dagger} *_{N} \mathcal{E} *_{M} \mathcal{D}^{\dagger}-\mathcal{S}_{1}^{\dagger} *_{N} \mathcal{S}_{1} *_{N} \mathcal{C}^{\dagger} *_{N} \mathcal{E} *_{M} \mathcal{N}_{1}^{\dagger}, \\
& \mathcal{A}_{1}=\mathcal{R}_{\mathcal{A}_{11} *_{N}} \mathcal{L}_{\mathcal{M}_{1}}, \mathcal{B}_{1}=\mathcal{R}_{\mathcal{N}_{1} *_{N}} \mathcal{L}_{\mathcal{B}_{11}}, \mathcal{C}_{1}=\mathcal{R}_{\mathcal{A}_{11} *_{N}}\left(\mathcal{R}_{\mathcal{N}_{1}}\right)^{\eta *}, \mathcal{D}_{1}=\left(\mathcal{L}_{\mathcal{M}_{1}}\right)^{\eta *} *_{N} \mathcal{L}_{\mathcal{B}_{11}} \text {, } \\
& \mathcal{E}_{1}=\mathcal{R}_{\mathcal{A}_{11} *_{N}} \mathcal{E}_{11} *_{N} \mathcal{L}_{\mathcal{B}_{11}}, \quad \mathcal{M}=\mathcal{R}_{\mathcal{A}_{1} *_{N}} \mathcal{C}_{1}, \quad \mathcal{N}=\mathcal{D}_{1} *_{N} \mathcal{L}_{\mathcal{B}_{1}}, \quad \mathcal{S}=\mathcal{C}_{1} *_{N} \mathcal{L}_{\mathcal{M}} .
\end{aligned}
$$

Theorem 4.1. Let $\mathcal{A}, \mathcal{B}, \mathcal{C}, \mathcal{D}, \mathcal{E}$ be given quaternion tensors mentioned above. The equation (1.1) is consistent if and only if

$$
\begin{aligned}
& \mathcal{R}_{\mathcal{A}} *_{N} \mathcal{E} *_{M} \mathcal{L}_{\mathcal{D}}=0, \quad \mathcal{R}_{\mathcal{C}} *_{N} \mathcal{E} *_{M} \mathcal{L}_{\mathcal{B}}=0, \quad \mathcal{R}_{\mathcal{S}_{1} *_{N}} \mathcal{R}_{\mathcal{A}} *_{N} \mathcal{E}=0, \\
& \mathcal{E} *_{M} \mathcal{L}_{\mathcal{B}} *_{M} \mathcal{L}_{\mathcal{N}_{1}}=0, \quad \mathcal{R}_{\mathcal{S}_{2}} *_{M}\left(\mathcal{L}_{\mathcal{B}}\right)^{\eta *} *_{M} \mathcal{E}^{\eta *}=0 \\
& \mathcal{E}^{\eta *} *_{N}\left(\mathcal{L}_{\mathcal{A}}\right)^{\eta *} *_{N}\left(\mathcal{L}_{\mathcal{N}_{1}}\right)^{\eta *}=0, \quad \mathcal{R}_{\mathcal{M}} *_{N} \mathcal{R}_{\mathcal{A}_{1}} *_{N} \mathcal{E}_{1}=0 \\
& \mathcal{E}_{1} *_{N} \mathcal{L}_{\mathcal{B}_{1}} *_{N} \mathcal{L}_{\mathcal{N}}=0, \quad \mathcal{R}_{\mathcal{A}_{1}} *_{N} \mathcal{E}_{1} *_{N} \mathcal{L}_{\mathcal{D}_{1}}=0, \quad \mathcal{R}_{\mathcal{C}_{1} *_{N}} \mathcal{E}_{1} *_{N} \mathcal{L}_{\mathcal{B}_{1}}=0 .
\end{aligned}
$$

In this case, the $\eta$-Hermitian solution to the equation (1.1) can be expressed as

$$
\mathcal{X}=\frac{\mathcal{X}_{1}+\mathcal{X}_{2}^{\eta *}}{2}, \quad \mathcal{Y}=\frac{\mathcal{Y}_{1}+\mathcal{Y}_{1}^{\eta *}}{2}
$$

where

$$
\begin{aligned}
& \mathcal{X}_{1}=\mathcal{A}^{\dagger} *_{N} \mathcal{E} *_{M} \mathcal{B}^{\dagger}-\mathcal{A}^{\dagger} *_{N} \mathcal{S}_{1} *_{N} \mathcal{C}^{\dagger} *_{N} \mathcal{E} *_{M} \mathcal{N}_{1}^{\dagger} *_{N} \mathcal{D} *_{M} \mathcal{B}^{\dagger} \\
& -\mathcal{A}^{\dagger} *_{N} \mathcal{C} *_{N} \mathcal{M}_{1}^{\dagger} *_{N} \mathcal{E} *_{M} \mathcal{B}^{\dagger}-\mathcal{A}^{\dagger} *_{N} \mathcal{S}_{1} *_{N} \mathcal{V}_{2} *_{M} \mathcal{R}_{\mathcal{N}_{1}} *_{N} \mathcal{D} *_{M} \mathcal{B}^{\dagger} \\
& +\mathcal{L}_{\mathcal{A}} *_{N} \mathcal{V}_{4}+\mathcal{V}_{5} *_{M} \mathcal{R}_{\mathcal{B}}, \\
& \mathcal{X}_{2}=\left(\mathcal{B}^{\dagger}\right)^{\eta *} *_{M} \mathcal{E}^{\eta *} *_{N}\left(\mathcal{A}^{\dagger}\right)^{\eta *}-\left(\mathcal{B}^{\dagger}\right)^{\eta *} *_{M} \mathcal{D}^{\eta *} *_{N}\left(\mathcal{M}_{1}^{\dagger}\right)^{\eta *} *_{N} \mathcal{E}^{\eta *} *_{N}\left(\mathcal{A}^{\dagger}\right)^{\eta *}
\end{aligned}
$$




$$
\begin{aligned}
& -\left(\mathcal{B}^{\dagger}\right)^{\eta *} *_{M} \mathcal{S}_{2} *_{N}\left(\mathcal{D}^{\dagger}\right)^{\eta *} *_{M} \mathcal{E}^{\eta *} *_{N}\left(\mathcal{N}_{1}^{\dagger}\right)^{\eta *} *_{M} \mathcal{C}^{\eta *} *_{N}\left(\mathcal{A}^{\dagger}\right)^{\eta *}+\left(\mathcal{R}_{\mathcal{B}}\right)^{\eta *} *_{M} \mathcal{T}_{4} \\
& +\mathcal{T}_{5} *_{N}\left(\mathcal{L}_{\mathcal{A}}\right)^{\eta *}-\left(\mathcal{B}^{\dagger}\right)^{\eta *} *_{M} \mathcal{S}_{2} *_{N} \mathcal{T}_{2} *_{M}\left(\mathcal{L}_{\mathcal{N}_{1}}\right)^{\eta *} *_{M} \mathcal{C}^{\eta *} *_{N}\left(\mathcal{A}^{\dagger}\right)^{\eta *}, \\
& \mathcal{Y}_{1}=\mathcal{M}_{1}^{\dagger} *_{N} \mathcal{E} *_{M} \mathcal{D}^{\dagger}+\mathcal{S}_{1}^{\dagger} *_{N} \mathcal{S}_{1} *_{N} \mathcal{C}^{\dagger} *_{N} \mathcal{E} *_{M} \mathcal{N}_{1}^{\dagger}+\mathcal{L}_{\mathcal{M}_{1} *_{N}} \mathcal{L}_{\mathcal{S}_{1}} *_{N} \mathcal{V}_{1} \\
& +\mathcal{L}_{\mathcal{M}_{1}} *_{N} \mathcal{V}_{2} *_{N} \mathcal{R}_{\mathcal{N}_{1}}+\mathcal{V}_{3} *_{N} \mathcal{R}_{\mathcal{D}}
\end{aligned}
$$

or

$$
\begin{aligned}
\mathcal{Y}_{1}= & \left(\mathcal{N}_{1}^{\dagger}\right)^{\eta *} *_{M} \mathcal{E}^{\eta *} *_{N}\left(\mathcal{C}^{\dagger}\right)^{\eta *}+\mathcal{S}_{2}^{\dagger} *_{M} \mathcal{S}_{2} *_{N}\left(\mathcal{D}^{\dagger}\right)^{\eta *} *_{N} \mathcal{E}^{\eta *} *_{N}\left(\mathcal{M}_{1}^{\dagger}\right)^{\eta *} \\
& -\left(\mathcal{R}_{\mathcal{N}_{1}}\right)^{\eta *} *_{N} \mathcal{L}_{\mathcal{S}_{2} *_{N}} \mathcal{T}_{1}-\left(\mathcal{R}_{\mathcal{N}_{1}}\right)^{\eta *} *_{N} \mathcal{T}_{2} *_{N}\left(\mathcal{L}_{\mathcal{M}_{1}}\right)^{\eta *}-\mathcal{T}_{3} *_{N}\left(\mathcal{L}_{\mathcal{C}}\right)^{\eta *}
\end{aligned}
$$

and

$$
\begin{aligned}
& \mathcal{V}_{1}=\left(\begin{array}{ll}
\mathcal{I} & 0
\end{array}\right) *_{N}\left(\mathcal{A}_{11}^{\dagger} *_{N}\left(\mathcal{E}_{11}-\mathcal{L}_{\mathcal{M}_{1} *_{N}} \mathcal{V}_{2} *_{N} \mathcal{R}_{\mathcal{N}_{1}}-\left(\mathcal{R}_{\mathcal{N}_{1}}\right)^{\eta *} *_{N} \mathcal{T}_{2} *_{N}\left(\mathcal{L}_{\mathcal{M}_{1}}\right)^{\eta *}\right)\right. \\
& \left.+\mathcal{W}_{1} *_{N} \mathcal{B}_{11}+\mathcal{L}_{\mathcal{A}_{11}} *_{N} \mathcal{W}_{2}\right) \\
& \mathcal{V}_{2}=\mathcal{A}_{1}^{\dagger} *_{N} \mathcal{E}_{1} *_{M} \mathcal{B}_{1}^{\dagger}-\mathcal{A}_{1}^{\dagger} *_{N} \mathcal{S} *_{N} \mathcal{C}_{1}^{\dagger} *_{N} \mathcal{E}_{1} *_{N} \mathcal{N}^{\dagger} *_{N} \mathcal{D}_{1} *_{N} \mathcal{B}_{1}^{\dagger} \\
& -\mathcal{A}_{1}^{\dagger} *_{N} \mathcal{C}_{1} *_{N} \mathcal{M}^{\dagger} *_{N} \mathcal{E}_{1} *_{N} \mathcal{B}_{1}^{\dagger}-\mathcal{A}_{1}^{\dagger} *_{N} \mathcal{S} *_{N} \mathcal{W}_{4} *_{N} \mathcal{R}_{\mathcal{N}} *_{N} \mathcal{D}_{1} *_{N} \mathcal{B}_{1}^{\dagger} \\
& +\mathcal{L}_{\mathcal{A}_{1} *_{N}} \mathcal{W}_{5}+\mathcal{W}_{6} *_{N} \mathcal{R}_{\mathcal{B}_{1}} \\
& \mathcal{V}_{3}=\left(\mathcal{R}_{\mathcal{A}_{11} *_{N}}\left(\mathcal{E}_{11}-\mathcal{L}_{\mathcal{M}_{1} *_{N}} \mathcal{V}_{2} *_{N} \mathcal{R}_{\mathcal{N}_{1}}-\left(\mathcal{R}_{\mathcal{N}_{1}}\right)^{\eta *} *_{N} \mathcal{T}_{2} *_{N}\left(\mathcal{L}_{\mathcal{M}_{1}}\right)^{\eta *}\right) *_{N} \mathcal{B}_{11}^{\dagger}\right. \\
& \left.-\mathcal{A}_{11} *_{N} \mathcal{W}_{1}-\mathcal{W}_{3} *_{N} \mathcal{R}_{\mathcal{B}_{11}}\right) *_{N}\left(\begin{array}{l}
\mathcal{I} \\
0
\end{array}\right) \text {, } \\
& \mathcal{T}_{1}=\left(\begin{array}{ll}
0 & \mathcal{I}
\end{array}\right) *_{N}\left(\mathcal{A}_{11}^{\dagger} *_{N}\left(\mathcal{E}_{11}-\mathcal{L}_{\mathcal{M}_{1} *_{N}} \mathcal{V}_{2} *_{N} \mathcal{R}_{\mathcal{N}_{1}}-\left(\mathcal{R}_{\mathcal{N}_{1}}\right)^{\eta *} *_{N} \mathcal{T}_{2} *_{N}\left(\mathcal{L}_{\mathcal{M}_{1}}\right)^{\eta *}\right)\right. \\
& \left.+\mathcal{W}_{1} *_{N} \mathcal{B}_{11}+\mathcal{L}_{\mathcal{A}_{11}} *_{N} \mathcal{W}_{2}\right) \\
& \mathcal{T}_{2}=\mathcal{M}^{\dagger} *_{N} \mathcal{E}_{1} *_{M} \mathcal{D}_{1}^{\dagger}+\mathcal{S} *_{N} \mathcal{S}^{\dagger} *_{N} \mathcal{C}_{1}^{\dagger} *_{N} \mathcal{E}_{1} *_{N} \mathcal{N}^{\dagger}+\mathcal{L}_{\mathcal{M}} *_{N} \mathcal{L}_{\mathcal{S}} *_{N} \mathcal{W}_{7} \\
& +\mathcal{L}_{\mathcal{M}} *_{N} \mathcal{W}_{4} *_{N} \mathcal{R}_{\mathcal{N}}+\mathcal{W}_{8} *_{N} \mathcal{R}_{\mathcal{D}_{1}}, \\
& \mathcal{T}_{3}=\left(\mathcal{R}_{\mathcal{A}_{11} *_{N}}\left(\mathcal{E}_{11}-\mathcal{L}_{\mathcal{M}_{1}} *_{N} \mathcal{V}_{2} *_{N} \mathcal{R}_{\mathcal{N}_{1}}-\left(\mathcal{R}_{\mathcal{N}_{1}}\right)^{\eta *} *_{N} \mathcal{T}_{2} *_{N}\left(\mathcal{L}_{\mathcal{M}_{1}}\right)^{\eta *}\right) *_{N} \mathcal{B}_{11}^{\dagger}\right. \\
& \left.-\mathcal{A}_{11} *_{N} \mathcal{W}_{1}-\mathcal{W}_{3} *_{N} \mathcal{R}_{\mathcal{B}_{11}}\right) *_{N}\left(\begin{array}{l}
0 \\
\mathcal{I}
\end{array}\right) \text {, }
\end{aligned}
$$

and $\mathcal{V}_{4}, \mathcal{V}_{5}, \mathcal{T}_{4}, \mathcal{T}_{5}, \mathcal{W}_{1}, \ldots, \mathcal{W}_{8}$ are arbitrary tensors over $\mathbb{H}$ with appropriate sizes.

Proof. We prove that the equation (1.1) has a solution with $\mathcal{Y}$ being $\eta$-Hermitian if and only if the following system

$$
\begin{aligned}
\mathcal{A} *_{N} \mathcal{X}_{1} *_{M} \mathcal{B}+\mathcal{C} *_{N} \mathcal{Y}_{1} *_{N} \mathcal{D} & =\mathcal{E} \\
\mathcal{B}^{\eta *} *_{M} \mathcal{X}_{2} *_{N} \mathcal{A}^{\eta *}+\mathcal{D}^{\eta *} *_{N} \mathcal{Y}_{1} *_{N} \mathcal{C}^{\eta *} & =\mathcal{E}^{\eta *}
\end{aligned}
$$

has a solution. 
Firstly, if the equation (1.1) has a solution, denoted as $(\widetilde{\mathcal{X}}, \widetilde{\mathcal{Y}})$, then it is obvious that the system 4.1 has a solution shaped like $\left(\mathcal{X}_{1}, \mathcal{X}_{2}, \mathcal{Y}_{1}\right)=\left(\tilde{\mathcal{X}}, \tilde{\mathcal{X}} \eta^{*}, \widetilde{\mathcal{Y}}\right)$.

Next we show that if the system (4.1) has a solution, then the equation (1.1) is solvable. Assume that the system (4.1) has a solution $\left(\mathcal{X}_{1}, \mathcal{X}_{2}, \mathcal{Y}_{1}\right)$. We will prove that

$$
(\mathcal{X}, \mathcal{Y})=\left(\frac{\mathcal{X}_{1}+\mathcal{X}_{2}^{\eta *}}{2}, \frac{\mathcal{Y}_{1}+\mathcal{Y}_{1}^{\eta *}}{2}\right)
$$

is a solution to the equation (1.1).

Substituting 4.2 into the equation 1.1) yields

$$
\begin{aligned}
& \mathcal{A} *_{N}\left(\frac{\mathcal{X}_{1}+\mathcal{X}_{2}^{\eta *}}{2}\right) *_{M} \mathcal{B}+\mathcal{C} *_{N}\left(\frac{\mathcal{Y}_{1}+\mathcal{Y}_{1}^{\eta *}}{2}\right) *_{N} \mathcal{D} \\
= & \frac{1}{2} \mathcal{A} *_{N} \mathcal{X}_{1} *_{M} \mathcal{B}+\frac{1}{2} \mathcal{C} *_{N} \mathcal{Y}_{1} *_{N} \mathcal{D}+\frac{1}{2} \mathcal{A} *_{N} \mathcal{X}_{2}^{\eta *} *_{M} \mathcal{B}+\frac{1}{2} \mathcal{C} *_{N} \mathcal{Y}_{1}^{\eta *} *_{M} \mathcal{D} \\
= & \frac{1}{2}\left(\mathcal{A} *_{N} \mathcal{X}_{1} *_{M} \mathcal{B}+\mathcal{C} *_{N} \mathcal{Y}_{1} *_{N} \mathcal{D}\right)+\frac{1}{2}\left(\mathcal{B}^{\eta *} *_{M} \mathcal{X}_{2} *_{N} \mathcal{A}^{\eta *}+\mathcal{D}^{\eta *} *_{N} \mathcal{Y}_{1} *_{N} \mathcal{C}^{\eta *}\right)^{\eta *} \\
= & \mathcal{E} .
\end{aligned}
$$

This implies that 4.2 is a solution to the equation 1.1 . We can get the solvability conditions and the expression of the general $\eta$-Hermitian solution to the equation (1.1) by Theorem 3.1 .

Remark 4.2. We can also present the solvability conditions and an expression of a solution with $\mathcal{X}$ being $\eta$-Hermitian to the tensor equation over $\mathbb{H}$ :

$$
\mathcal{A} *_{N} \mathcal{X} *_{N} \mathcal{B}+\mathcal{C} *_{N} \mathcal{Y} *_{M} \mathcal{D}=\mathcal{E}
$$

where $\mathcal{A}, \mathcal{B}, \mathcal{C}, \mathcal{D}, \mathcal{E}$ are given, $\mathcal{X}$ and $\mathcal{Y}$ are unknown.

Now we turn our attention to consider the solvability conditions and the general $\eta$-Hermitian solution to the system (1.3) where $\mathcal{X}_{1}, \mathcal{X}_{2}, \mathcal{Y}_{1}$ are unknowns and $\mathcal{A}_{1} \in$ $\mathbb{H}^{I_{1} \times \cdots \times I_{N} \times J_{1} \times \cdots \times J_{N}}, \mathcal{A}_{2} \in \mathbb{H}^{I_{1} \times \cdots \times I_{N} \times S_{1} \times \cdots \times S_{N}}, \mathcal{B}_{1} \in \mathbb{H}^{I_{1} \times \cdots \times I_{N} \times K_{1} \times \cdots \times K_{N}}, \mathcal{B}_{2} \in$ $\mathbb{H}^{I_{1} \times \cdots \times I_{N} \times K_{1} \times \cdots \times K_{N}}, \mathcal{E}_{i} \in \mathbb{H}^{I_{1} \times \cdots \times I_{N} \times I_{1} \times \cdots \times I_{N}}$ are given quaternion tensors, and $\mathcal{E}_{i}(i=$ $1,2)$ are $\eta$-Hermitian. For simplicity, we set

$$
\begin{aligned}
& \mathcal{M}_{1}=\mathcal{R}_{\mathcal{A}_{1}} *_{N} \mathcal{B}_{1}, \quad \mathcal{M}_{2}=\mathcal{R}_{\mathcal{A}_{2} *_{N}} \mathcal{B}_{2}, \quad \mathcal{S}_{1}=\mathcal{B}_{1} *_{N} \mathcal{L}_{\mathcal{M}_{1}}, \quad \mathcal{S}_{2}=\mathcal{B}_{2} *_{N} \mathcal{L}_{\mathcal{M}_{2}} \\
& \mathcal{A}_{11}=\left(\begin{array}{llll}
\mathcal{L}_{\mathcal{M}_{1}} *_{N} & \mathcal{L}_{\mathcal{S}_{1}} & \mathcal{L}_{\mathcal{M}_{2}} *{ }_{N} & \mathcal{L}_{\mathcal{S}_{2}}
\end{array}\right), \quad \mathcal{B}_{11}=\left(\begin{array}{l}
\left(\mathcal{L}_{\mathcal{B}_{1}}\right)^{\eta *} \\
\left(\mathcal{L}_{\mathcal{B}_{2}}\right)^{\eta *}
\end{array}\right), \\
& \mathcal{E}_{11}=\mathcal{M}_{2}^{\dagger} *_{N} \mathcal{E}_{2} *_{N}\left(\mathcal{B}_{2}^{\dagger}\right)^{\eta *}+\mathcal{S}_{2}^{\dagger} *_{N} \mathcal{S}_{2} *_{N} \mathcal{B}_{2}^{\dagger} *_{N} \mathcal{E}_{2} *_{N}\left(\mathcal{M}_{2}^{\dagger}\right)^{\eta *} \\
& -\mathcal{M}_{1}^{\dagger} *_{N} \mathcal{E}_{1} *_{N}\left(\mathcal{B}_{1}^{\dagger}\right)^{\eta *}-\mathcal{S}_{1}^{\dagger} *_{N} \mathcal{S}_{1} *_{N} \mathcal{B}_{1}^{\dagger} *_{N} \mathcal{E}_{1} *_{N}\left(\mathcal{M}_{1}^{\dagger}\right)^{\eta *} \\
& \mathcal{A}=\mathcal{R}_{\mathcal{A}_{11} *_{N}} \mathcal{L}_{\mathcal{M}_{1}}, \mathcal{B}=\left(\mathcal{L}_{\mathcal{M}_{1}}\right)^{\eta *} *_{N} \mathcal{L}_{\mathcal{B}_{11}}, \mathcal{C}=\mathcal{R}_{\mathcal{A}_{11} *_{N}} \mathcal{L}_{\mathcal{M}_{2}}, \mathcal{D}=\left(\mathcal{L}_{\mathcal{M}_{2}}\right)^{\eta *} *_{N} \mathcal{L}_{\mathcal{B}_{11}},
\end{aligned}
$$

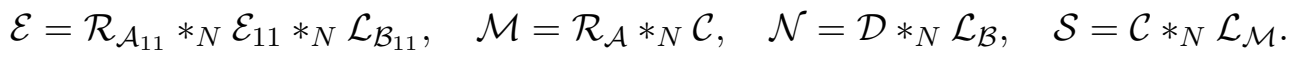


Theorem 4.3. Let $\mathcal{A}_{i}, \mathcal{B}_{i}, \mathcal{E}_{i}(i=1,2)$ be given quaternion tensors. Then the system (1.3) is consistent if and only if

$$
\begin{aligned}
& \mathcal{R}_{\mathcal{A}_{1} *_{N}} \mathcal{E}_{1} *_{N}\left(\mathcal{R}_{\mathcal{B}_{1}}\right)^{\eta *}=0, \quad \mathcal{R}_{\mathcal{M}_{1} *_{N}} \mathcal{R}_{\mathcal{A}_{1} *_{N}} \mathcal{E}_{1}=0 \\
& \mathcal{R}_{\mathcal{A}_{2} *_{N}} \mathcal{E}_{2} *_{N}\left(\mathcal{R}_{\mathcal{B}_{2}}\right)^{\eta *}=0, \quad \mathcal{R}_{\mathcal{M}_{2} *_{N}} \mathcal{R}_{\mathcal{A}_{2} *_{N}} \mathcal{E}_{2}=0, \quad \mathcal{R}_{\mathcal{M}} *_{N} \mathcal{R}_{\mathcal{A}} *_{N} \mathcal{E}=0, \\
& \mathcal{E} *_{N} \mathcal{L}_{\mathcal{B}} *_{N} \mathcal{L}_{\mathcal{N}}=0, \quad \mathcal{R}_{\mathcal{A}} *_{N} \mathcal{E} *_{N} \mathcal{L}_{\mathcal{D}}=0, \quad \mathcal{R}_{\mathcal{C}} *_{N} \mathcal{E} *_{N} \mathcal{L}_{\mathcal{B}}=0
\end{aligned}
$$

In this case, the expression of $\eta$-Hermitian solution $\left(\mathcal{X}_{1}, \mathcal{X}_{2}, \mathcal{Y}_{1}\right)$ can be deduced from (3.8)(3.11) and (3.12a) -3.12f) in Theorem 3.1.

Proof. We show that the system (1.3) has an $\eta$-Hermitian solution if and only if the system of quaternion tensor equations

$$
\begin{aligned}
& \mathcal{A}_{1} *_{N} \mathcal{X} *_{N} \mathcal{A}_{1}^{\eta^{*}}+\mathcal{B}_{1} *_{N} \mathcal{Y} *_{N} \mathcal{B}_{1}^{\eta^{*}}=\mathcal{E}_{1}, \\
& \mathcal{A}_{2} *_{N} \mathcal{Z} *_{N} \mathcal{A}_{2}^{\eta^{*}}+\mathcal{B}_{2} *_{N} \mathcal{Y} *_{N} \mathcal{B}_{2}^{\eta^{*}}=\mathcal{E}_{2}
\end{aligned}
$$

has a solution, where $\mathcal{E}_{i}(i=1,2)$ are $\eta$-Hermitian quaternion tensors. If the system (1.3) has an $\eta$-Hermitian solution, i.e., $\left(\mathcal{X}_{1}, \mathcal{X}_{2}, \mathcal{Y}_{1}\right)$, then the system 4.3 obviously has a solution $(\mathcal{X}, \mathcal{Y}, \mathcal{Z})=\left(\mathcal{X}_{1}, \mathcal{Y}_{1}, \mathcal{X}_{2}\right)$.

Conversely, if the system $(4.3)$ has a solution $(\mathcal{X}, \mathcal{Y}, \mathcal{Z})$, then

$$
\left(\mathcal{X}_{1}, \mathcal{X}_{2}, \mathcal{Y}_{1}\right)=\left(\frac{\mathcal{X}+\mathcal{X}^{\eta *}}{2}, \frac{\mathcal{Z}+\mathcal{Z}^{\eta *}}{2}, \frac{\mathcal{Y}+\mathcal{Y}^{\eta *}}{2}\right)
$$

is an $\eta$-Hermitian solution to the system $(1.3)$. Therefore we can get the solvability conditions by Theorem 3.1. And the expression of the $\eta$-Hermitian solution to the system (1.3) can be obtained from $(3.8)-(3.11)$ and $(3.12 \mathrm{a})-(3.12 \mathrm{f})$ when the conditions are met.

\section{Conclusion}

In this paper, we have established some necessary and sufficient conditions for the existence of the general solution to the system $(1.2)$ and constructed an expression of the general solution to the system when it is solvable in Theorem 3.1. We have provided an example to illustrate our main results. As applications of the system (1.2), we have given some necessary and sufficient conditions for the existence of an $\eta$-Hermitian solution to the two-sided Sylvester-type tensor equation (1.1) and the system (1.3). Moreover, we have presented expressions of such solutions when the solvability conditions are met.

\section{Acknowledgments}

The authors are grateful to an anonymous referee for useful comments and suggestions. This research is supported by the National Natural Science Foundation of China under grant no. 11971294. 


\section{References}

[1] A. D. Barbour and S. Utev, Solving the Stein equation in compound Poisson approximation, Adv. in Appl. Probab. 30 (1998), no. 2, 449-475.

[2] C. Bauckhage, Robust tensor classifiers for color object recognition, in: Image Analysis and Recognition, 352-363, Lecture Notes in Computer Science 4633, 2007.

[3] S. Brahma and B. Datta, An optimization approach for minimum norm and robust partial quadratic eigenvalue assignment problems for vibrating structures, J. Sound Vibration 324 (2009), no. 3-5, 471-489.

[4] Z. Chen and L. Lu, A projection method and Kronecker product preconditioner for solving Sylvester tensor equations, Sci. China Math. 55 (2012), no. 6, 1281-1292.

[5] M. Dehghan and M. Hajarian, Analysis of an iterative algorithm to solve the generalized coupled Sylvester matrix equations, Appl. Math. Model. 35 (2011), no. 7, 3285-3300.

[6] L. De Lathlauwer, A survey of tensor methods, 2009 IEEE International Symposium on Circuits and Systems, (2009), 2773-2776.

[7] S. De Leo and G. Scolarici, Right eigenvalue equation in quaternionic quantum mechanics, J. Phys. A 33 (2000), no. 15, 2971-2995.

[8] A. Einstein, The foundation of the general theory of relativity, in: The Collected Papers of Albert Einstein, Vol. 6: The Berlin years: writings, 1914-1917, 146-200, Princeton University Press, 1997.

[9] J. M. Fernandez and W. A. Schneeberger, Quaternionic computing, arXiv:quantph/0307017v2, (2004).

[10] L. Grasedyck, Existence and computation of low Kronecker-rank approximations for large linear systems of tensor product structure, Computing 72 (2004), no. 3-4, 247265.

[11] Y. Guan and D. Chu, Numerical computation for orthogonal low-rank approximation of tensors, SIAM J. Matrix Anal. Appl. 40 (2019), no. 3, 1047-1065.

[12] Y. Guan, M. T. Chu and D. Chu, Convergence analysis of an SVD-based algorithm for the best rank-1 tensor approximation, Linear Algebra Appl. 555 (2018), 53-69.

[13] _ SVD-based algorithms for the best rank-1 approximation of a symmetric tensor, SIAM J. Matrix Anal. Appl. 39 (2018), no. 3, 1095-1115. 
[14] W. R. Hamilton, Elements of Quaternions, Longmans Green and Co London, 1866.

[15] Z.-H. He, The general solution to a system of coupled Sylvester-type quaternion tensor equations involving $\eta$-Hermicity, Bull. Iranian Math. Soc. 45 (2019), no. 5, 1407-1430.

[16] Z.-H. He, O. M. Agudelo, Q.-W. Wang and B. De Moor, Two-sided coupled generalized Sylvester matrix equations solving using a simultaneous decomposition for fifteen matrices, Linear Algebra Appl. 496 (2016), 549-593.

[17] Z.-H. He, C. Navasca and Q.-W. Wang, Tensor decompositions and tensor equations over quaternion algebra, arXiv:1710.07552.

[18] Z.-H. He and Q.-W. Wang, A real quaternion matrix equation with applications, Linear Multilinear Algebra 61 (2013), no. 6, 725-740.

[19] Z.-H. He, Q.-W. Wang and Y. Zhang, Simultaneous decomposition of quaternion matrices involving $\eta$-Hermicity with applications, Appl. Math. Comput. 298 (2017), 13-35.

[20] A. Klein and P. Spreij, On Fisher's information matrix of an ARMAX process and Sylvester's resultant matrices, Linear Algebra Appl. 237/238 (1996), 579-590.

[21] T. Levi-Civita, The Absolute Differential Calculus: Calculus of tensors, Dover Phoenix Editions, Dover Publications, Mineola, NY, 2005.

[22] B.-W. Li, S. Tian, Y.-S. Sun and Z.-M. Hu, Schur-decomposition for 3D matrix equations and its application in solving radiative discrete ordinates equations discretized by Chebyshev collocation spectral method, J. Comput. Phys. 229 (2010), no. 4, 11981212.

[23] T. Li, Q.-W. Wang and X.-F. Duan, Numerical algorithms for solving discrete Lyapunov tensor equation, J. Comput. Appl. Math. 370 (2020), 112676, 11 pp.

[24] N. Liu, B. Zhang, J. Yan, Z. Chen, W. Liu, F. Bai and L. Chien, Text representation: From vector to tensor, Fifth IEEE International Conference on Data Mining, (2005), $725-728$.

[25] C. Navasca, L. De Lathauwer and S. Kindermann, Swamp reducing technique for tensor decomposition, 16th European Signal Processing Conference, (2008).

[26] L. Qi, H. Chen and Y. Chen, Tensor Eigenvalues and Their Applications, Advances in Mechanics and Mathematics 39, Springer, Singapore, 2018. 
[27] L. Qi and Z. Luo, Tensor Analysis: Spectral theory and special tensors, Other Titles in Applied Mathematics, SIAM, Philadelphia, 2017.

[28] L. Rodman, Topics in Quaternion Linear Algebra, Princeton Series in Applied Mathematics, Princeton University Press, Princeton, NJ, 2014.

[29] A. Shashua and T. Hazan, Non-negative tensor factorization with applications to statistics and computer vision, Proceeding of the 22nd international conference on Machine learning, (2005), 792-799.

[30] L. Sun, B. Zheng, C. Bu and Y. Wei, Moore-Penrose inverse of tensors via Einstein product, Linear Multilinear Algebra 64 (2016), no. 4, 686-698.

[31] C. C. Took and D. P. Mandic, Quaternion-valued stochastic gradient-based adaptive IIR filtering, IEEE Trans. Signal Process. 58 (2010), no. 7, 3895-3901.

[32] _ Augmented second-order statistics of quaternion random signals, Signal Process. 91 (2011), no. 2, 214-224.

[33] A. Varga, Robust pole assignment via Sylvester equation based state feedback parametrization, Proceedings of the 2000 IEEE International Symposium on Computer-Aided Control System Design, Anchorage, Alaska, (2000), 13-18.

[34] Q.-W. Wang and Z.-H. He, Solvability conditions and general solution for mixed Sylvester equations, Automatica J. IFAC 49 (2013), no. 9, 2713-2719.

[35] Q.-W. Wang, Z.-H. He and Y. Zhang, Constrained two-sided coupled Sylvester-type quaternion matrix equations, Automatica J. IFAC 101 (2019), 207-213.

[36] Q.-W. Wang and X. Xu, Iterative algorithms for solving some tensor equations, Linear Multilinear Algebra 67 (2019), no. 7, 1325-1349.

[37] Q.-W. Wang, X. Xu and X. Duan, Least squares solution of the quaternion Sylvester tensor equation, Accepted in Linear Multilinear Algebra, 2019.

[38] X. Xu and Q.-W. Wang, Extending BiCG and BiCR methods to solve the Stein tensor equation, Comput. Math. Appl. 77 (2019), no. 12, 3117-3127.

[39] F. Zhang, Quaternions and matrices of quaternions, Linear Algebra Appl. 251 (1997), $21-57$.

[40] Y. Zhang, D. Jiang and J. Wang, A recurrent neural network for solving Sylvester equation with time-varying coefficients, IEEE Trans. Neural Networks 13 (2002), no. 5, 1053-1063. 
Qing-Wen Wang and Xiao Wang

Department of Mathematics, Shanghai University, Shanghai 200444, China

E-mail address: wqw@t.shu.edu.cn, wqwshu9@126.com 